\title{
Chemical Analysis of the Ultrafaint Dwarf Galaxy Grus II. Signature of High-mass Stellar Nucleosynthesis*
}

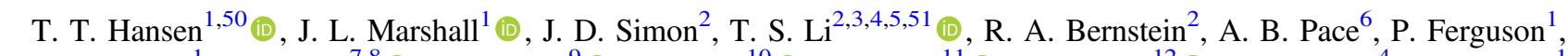

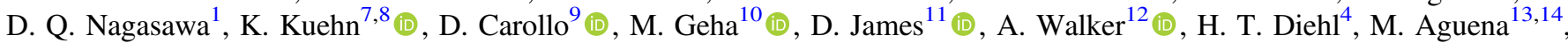
S. Allam ${ }^{4,15}$, S. Avila ${ }^{16}$, E. Bertin ${ }^{17,18}$, D. Brooks ${ }^{19}$ (i), E. Buckley-Geer ${ }^{4}$, D. L. Burke ${ }^{20,21}$ (1) , A. Carnero Rosell ${ }^{14,22}$, M. Carrasco Kind ${ }^{23,24}$ (1) J. Carretero ${ }^{25}$, M. Costanzi ${ }^{26,27}$, L. N. Da Costa ${ }^{14,28}$, S. Desai ${ }^{29}$, J. De Vicente ${ }^{22}$, P. Doel ${ }^{19}$, K. Eckert $^{30}{ }^{(1)}$, T. F. Eifler ${ }^{31,32}$, S. Everett ${ }^{33}$, I. Ferrero ${ }^{34}$, J. Frieman ${ }^{4,5}$, J. García-Bellido ${ }^{16}$ (1) E. Gaztanaga ${ }^{35,36}$, D. W. Gerdes ${ }^{37,38}$ (1), D. Gruen ${ }^{20,21,39}$ (1) , R. A. Gruendl ${ }^{23,24}$ (1) , J. Gschwend ${ }^{14,28}$, G. Gutierrez ${ }^{4}$, S. R. Hinton ${ }^{15}$ (1), D. L. Hollowood ${ }^{33}$ (1), K. Honscheid ${ }^{40,41}$, N. Kuropatkin ${ }^{4,19}$, M. A. G. Maia ${ }^{14,28}$, M. March $^{30}$, R. Miquel ${ }^{25,42}$ (1), A. Palmese ${ }^{4,5}$ (1), F. Paz-Chinchón ${ }^{23,24}$ (1),

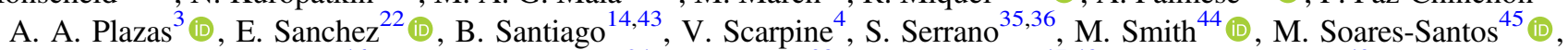
E. Suchyta ${ }^{46}$, M. E. C. Swanson ${ }^{24}$ (1) G. Tarle ${ }^{38}$, T. N. Varga ${ }^{47,48}$, and R. Wilkinson ${ }^{49}$ (DES Collaboration)

${ }^{1}$ Mitchell Institute for Fundamental Physics and Astronomy and Department of Physics and Astronomy, Texas A\&M University, College Station, TX 77843-4242, \author{
USA; thansen@tamu.edu \\ ${ }^{2}$ Carnegie Institution for Science, 813 Santa Barbara St., Pasadena, CA 91101, USA \\ ${ }^{3}$ Department of Astrophysical Sciences, Princeton University, Peyton Hall, Princeton, NJ 08544, USA \\ ${ }^{4}$ Fermi National Accelerator Laboratory, P.O. Box 500, Batavia, IL 60510, USA \\ ${ }^{5}$ Kavli Institute for Cosmological Physics, University of Chicago, Chicago, IL 60637, USA \\ ${ }^{6}$ McWilliams Center for Cosmology, Carnegie Mellon University, 5000 Forbes Ave, Pittsburgh, PA 15213, USA \\ ${ }^{7}$ Australian Astronomical Optics, Macquarie University, North Ryde, NSW 2113, Australia \\ ${ }^{8}$ Lowell Observatory, 1400 W Mars Hill Rd., Flagstaff, AZ 86001, USA \\ ${ }^{9}$ INAF-Osservatorio Astronomico di Torino-Strada Osservatorio 20, Pino Torinese, I-10020, Italy \\ ${ }_{11}^{10}$ Department of Astronomy, Yale University, New Haven, CT 06520, USA \\ ${ }^{11}$ Harvard-Smithsonian Center for Astrophysics, Cambridge, MA 02138, USA \\ ${ }^{12}$ Cerro Tololo Inter-American Observatory, National Optical Astronomy Observatory, Casilla 603, La Serena, Chile \\ ${ }^{13}$ Departamento de Física Matemática, Instituto de Física, Universidade de São Paulo, CP 66318, São Paulo, SP, 05314-970, Brazil \\ ${ }^{15}$ School of Mathematics and Physics, University of Queensland, Brisbane, QLD 4072, Australia \\ ${ }^{16}$ Instituto de Fisica Teorica UAM/CSIC, Universidad Autonoma de Madrid, E-28049 Madrid, Spain \\ ${ }^{17}$ CNRS, UMR 7095, Institut d'Astrophysique de Paris, F-75014, Paris, France \\ ${ }^{18}$ Sorbonne Universités, UPMC Univ Paris 06, UMR 7095, Institut d'Astrophysique de Paris, F-75014, Paris, France \\ ${ }_{19}$ Department of Physics \& Astronomy, University College London, Gower Street, London, WC1E 6BT, UK \\ ${ }^{20}$ Kavli Institute for Particle Astrophysics \& Cosmology, P.O. Box 2450, Stanford University, Stanford, CA 94305, USA \\ ${ }^{21}$ SLAC National Accelerator Laboratory, Menlo Park, CA 94025, USA \\ ${ }^{22}$ Centro de Investigaciones Energéticas, Medioambientales y Tecnológicas (CIEMAT), Madrid, Spain \\ ${ }^{23}$ Department of Astronomy, University of Illinois at Urbana-Champaign, 1002 W. Green St., Urbana, IL 61801, USA \\ ${ }^{24}$ National Center for Supercomputing Applications, 1205 West Clark St., Urbana, IL 61801, USA \\ ${ }^{25}$ Institut de Física d'Altes Energies (IFAE), The Barcelona Institute of Science and Technology, Campus UAB, E-08193 Bellaterra (Barcelona), Spain \\ ${ }^{26}$ INAF-Osservatorio Astronomico di Trieste, via G.B. Tiepolo 11, I-34143 Trieste, Italy \\ ${ }^{27}$ Institute for Fundamental Physics of the Universe, Via Beirut 2, I-34014 Trieste, Italy \\ ${ }^{28}$ Observatório Nacional, Rua Gal. José Cristino 77, Rio de Janeiro, RJ-20921-400, Brazil \\ ${ }^{30}$ Department of Physics and Astronomy, University of Pennsylvania, Philadelphia, PA 19104, USA \\ ${ }^{31}$ Department of Astronomy/Steward Observatory, University of Arizona, 933 North Cherry Ave., Tucson, AZ 85721-0065, USA \\ 32 Jet Propulsion Laboratory, California Institute of Technology, 4800 Oak Grove Dr., Pasadena, CA 91109, USA \\ ${ }^{33}$ Santa Cruz Institute for Particle Physics, Santa Cruz, CA 95064, USA \\ ${ }^{34}$ Institute of Theoretical Astrophysics, University of Oslo, P.O. Box 1029 Blindern, NO-0315 Oslo, Norway \\ ${ }^{35}$ Institut d'Estudis Espacials de Catalunya (IEEC), E-08034 Barcelona, Spain \\ ${ }^{36}$ Institute of Space Sciences (ICE, CSIC), Campus UAB, Carrer de Can Magrans, s/n, E-08193 Barcelona, Spain \\ ${ }^{37}$ Department of Astronomy, University of Michigan, Ann Arbor, MI 48109, USA \\ ${ }^{38}$ Department of Physics, University of Michigan, Ann Arbor, MI 48109, USA \\ ${ }^{39}$ Department of Physics, Stanford University, 382 Via Pueblo Mall, Stanford, CA 94305, USA \\ ${ }^{40}$ Center for Cosmology and Astro-Particle Physics, The Ohio State University, Columbus, OH 43210, USA \\ ${ }^{41}$ Department of Physics, The Ohio State University, Columbus, OH 43210, USA \\ ${ }^{42}$ Institució Catalana de Recerca i Estudis Avançats, E-08010 Barcelona, Spain \\ ${ }^{43}$ Instituto de Física, UFRGS, Caixa Postal 15051, Porto Alegre, RS-91501-970, Brazil \\ ${ }^{44}$ School of Physics and Astronomy, University of Southampton, Southampton, SO17 1BJ, UK \\ ${ }^{46}$ Computer Science and Mathematics Division, Oak Ridge National Laboratory, Oak Ridge, TN 37831, USA \\ 47 Max Planck Institute for Extraterrestrial Physics, Giessenbachstrasse, D-85748 Garching, Germany
${ }^{48}$ Universitäts-Sternwarte, Fakultät für Physik, Ludwig-Maximilians Universität München, Scheinerstr. 1, D-81679 München, Germany \\ ${ }^{49}$ Department of Physics and Astronomy, Pevensey Building, University of Sussex, Brighton, BN1 9QH, UK \\ Received 2020 January 2; revised 2020 May 12; accepted 2020 May 19; published 2020 July 16
}

\footnotetext{
${ }_{50}^{*}$ This paper includes data gathered with the $6.5 \mathrm{~m}$ Magellan Telescopes located at Las Campanas Observatory, Chile.

${ }_{51}^{50}$ Mitchell Astronomy Fellow.

51 NHFP Einstein Fellow.
} 


\begin{abstract}
We present a detailed abundance analysis of the three brightest member stars at the top of the giant branch of the ultrafaint dwarf (UFD) galaxy Grus II. All stars exhibit a higher than expected $[\mathrm{Mg} / \mathrm{Ca}]$ ratio compared to metalpoor stars in other UFD galaxies and in the Milky Way (MW) halo. Nucleosynthesis in high-mass $\left(\geqslant 20 M_{\odot}\right)$ corecollapse supernovae has been shown to create this signature. The abundances of this small sample (three) stars suggests the chemical enrichment of Grus II could have occurred through substantial high-mass stellar evolution, and is consistent with the framework of a top-heavy initial mass function. However, with only three stars it cannot be ruled out that the abundance pattern is the result of a stochastic chemical enrichment at early times in the galaxy. The most metal-rich of the three stars also possesses a small enhancement in rapid neutron-capture ( $r$-process) elements. The abundance pattern of the $r$-process elements in this star matches the scaled $r$-process pattern of the solar system and $r$-process enhanced stars in other dwarf galaxies and in the MW halo, hinting at a common origin for these elements across a range of environments. All current proposed astrophysical sites of $r$-process element production are associated with high-mass stars, thus the possible top-heavy initial mass function of Grus II would increase the likelihood of any of these events occurring. The time delay between the $\alpha$ and $r$-process element enrichment of the galaxy favors a neutron star merger as the origin of the $r$-process elements in Grus II.
\end{abstract}

Unified Astronomy Thesaurus concepts: Chemical abundances (224); Dwarf galaxies (416); Chemically peculiar stars (226); Stellar abundances (1577)

Supporting material: machine-readable tables

\section{Introduction}

Recent exploration of the chemical abundances in ultrafaint dwarf (UFD) galaxies have revealed abundance patterns of these objects similar to those that have been detected in the majority of metal-poor Milky Way (MW) halo stars (Frebel \& Norris 2015). However, some notable outliers have also been observed, for example Reticulum II (Ret II) and Tucana III (Tuc III) both show enhancement in rapid neutron-capture ( $r$-process) elements ( $\mathrm{Ji}$ et al. 2016a; Roederer et al. 2016; Hansen et al. 2017; Marshall et al. 2019). This chemical signature is also seen in a very small fraction of MW halo stars (Barklem et al. 2005; Hansen et al. 2018). More rare abundance patterns have also been detected in the UFD galaxies Horologium I (Hor I) and Hercules (Her), with Hor I exhibiting a deficiency in $\alpha$ elements like $\mathrm{Mg}$ and $\mathrm{Ca}$ (Nagasawa et al. 2018 ), and some stars in Her showing very high $[\mathrm{Mg} / \mathrm{Ca}]$ ratios compared to the average metal-poor MW halo and UFD galaxy stars (Koch et al. 2008).

The UFD galaxies are small dark matter dominated systems that have slower chemical enrichment compared to the larger dwarf spheroidal galaxies and the MW (Simon 2019). Hence, the chemical abundances of the stars in these systems provide a window to study single nucleosynthesis events in closed environments. The peculiar chemical patterns described above are particularly important to study rare chemical enrichment events, such as neutron star mergers (NSMs) or special types of core-collapse supernovae (CCSNe) such as magnetorotational supernovae (Winteler et al. 2012), collapsars (Siegel et al. 2019), and supernovae with mixing and fallback (Umeda \& Nomoto 2003).

The chemistry of the first generation of low-mass stars to form in a galaxy is tightly linked to the mass range of the first population of massive stars (Population III) that formed and terminally evolved in them. Placco et al. (2016) used the abundance pattern of the most metal-poor MW halo stars along with nucleosynthesis models from Heger \& Woosley (2010) to constrain the mass range of the Population III stars responsible for the initial chemical enrichment of the Galaxy. Similar analysis in UFD galaxies are hampered by the small number of stars for which detailed chemical abundances can be determined in these systems.

On the other hand, UFD galaxies have been used to study the effect of environment on the initial stellar mass function (IMF). The long relaxation times of this type of galaxy means that the low-mass stellar IMF can be measured directly from the number of stars below the main-sequence turn-off present in the Galaxy today (Geha et al. 2013). The low luminosity of these systems, however, makes it difficult to obtain the photometric observations needed for this measurement. For those UFD galaxies where the measurements have been made (Boo I, CVn II, ComBer, Her, Leo IV, UMa I), evidence of variations of the IMF with environment have been found. For example Gennaro et al. (2018) measured the IMF for a sample of six UFDs and found that their subsolar stellar IMFs were generally more bottom-light than the IMF for MW disk stars, though with a large scatter within the sample. In another study Geha et al. (2013) measured the IMF for Hercules and Leo IV and also found bottom-light IMFs for these galaxies. For other types of galaxies, some correlations have been detected between IMFs and galaxy properties, with the largest galaxies having bottom-heavy IMFs and smaller galaxies having more bottom-light IMFs (Geha et al. 2013). This variation is also supported by the results from Kalirai et al. (2013), who measured the IMF of the Small Magellanic Cloud and found a shallower slope than what is determined in the MW.

It is important to note that even though the low-mass stellar populations of today's UFD galaxies provides a direct way to measure the IMF of these systems, this does not provide any information on the IMF of the higher stellar mass population previously present in the galaxy. However, here the chemistry of the metal-poor stars may provide some clues. The chemical abundances of the most metal-poor stars in the UFD galaxies constitute a record of the nucleosynthesis happening in the first generation of massive stars formed in these systems. Hence, the chemical abundance pattern can provide constraints on the mass range of the first stars to form in the UFD galaxy.

In this paper we analyze the UFD galaxy Grus II (Gru II). Gru II was identified as a candidate MW satellite galaxy in the 
Dark Energy Survey (DES) at a distance of $53 \mathrm{kpc}$ (DrlicaWagner et al. 2015). Spectroscopic follow-up of the system was presented by Simon et al. (2020), who were unable to resolve a velocity dispersion or detect a metallicity spread, indicative of dwarf galaxies, for the system. However, based on the large physical size $\left(r_{1 / 2}=94 \mathrm{pc}\right)$ and low metallicity $([\mathrm{Fe} / \mathrm{H}]=-2.51 \pm 0.11)$, Simon et al. (2020) classify the system as a likely dwarf galaxy. We present here a detailed chemical abundance analysis of the three brightest member stars at the top of the giant branch of Gru II. The paper is organized as follows: observations and analysis of the stars are described in Sections 2 and 3, Section 4 presents our results, which are then discussed in Section 5, and Section 6 provides a summary.

\section{Observations}

A total sample of five stars were observed with the MIKE spectrograph (Bernstein et al. 2003) at the Magellan-Clay Telescope at Las Campanas Observatory. Table 1 lists the targets, observing dates, and exposure times. The two brightest stars DES J220423.91-463702.5 and DES J220409.98-462102.2 (hereafter referred to as J220423 and J220409) were observed during a run in 2016 August. The first spectrum of DES J220352.01-462446.5 (J220352) was observed in 2017 August and the second in 2018 November along with spectra of DES J220318.62-464116.4 (J220318) and DES J220253.88463522.6 (J220253). Three of the stars (J220423, J220409, and J220352) were selected as spectroscopically confirmed members from medium-resolution velocity and metallicity measurements (Simon et al. 2020). The brightest star, J220423, was first identified as a member from an observing run with the AngloAustralia Telescope (AAT) in 2016 July. While the AAT observing run was mostly for targeting the Tucana III stream (see details in $\mathrm{Li}$ et al. 2018), a small fraction of time was also spent on bright candidate members in Gru II and Tucana IV during nights with cirrus cloud coverage, to identify bright member stars for MIKE observations. The membership of J220423 was subsequently confirmed with Magellan/IMACS spectroscopy (Simon et al. 2020). The remaining two stars, J220318 and J220253, were selected as member candidates from Pace \& Li (2019), although with relatively low membership probabilities ( $p_{i}=0.47$ and $p_{i}=0.14$, respectively). Radial velocities derived from short MIKE exposures establish these as nonmembers (see Table 1). Figure 1 shows a color-magnitude diagram of Gru II member stars; star symbols mark the five stars observed, filled symbols are members, while open symbols are not members.

The spectra of the stars were obtained using a $0 . / 7$ slit with $2 \times 2$ pixel binning resulting in a spectral resolution of $R=\lambda$ / $\Delta \lambda \approx 41,000$ in the blue and 32,000 in the red. The spectra cover $3310 \AA<\lambda<5000 \AA$ in the blue channel and $4830 \AA<\lambda<9160 \AA$ in the red. The data were reduced using the latest version of the CarPy MIKE pipeline (Kelson et al. 2000; Kelson 2003). For stars with multiple spectra taken on the same run (J220409, J220423, and J220318) these were co-added (summed) during reduction while the spectra from multiple runs (J220352) were coadded after reduction. Following reduction the spectra were normalized and shifted to rest wavelength. Final reduced and normalized spectra around the $\mathrm{Mg}$ I b lines, the Ba II $6141 \AA$ line, and the Eu II $4129 \AA$ line are shown in Figure 2. Signal-to-noise ratios $(\mathrm{S} / \mathrm{N})$ per pixel of the final reduced spectra at 4100 and $5500 \AA$ are listed in Table 1. Heliocentric radial velocities of the stars were determined by cross-correlation (Tonry \& Davis 1979) of the target spectra with a spectrum of the radial-velocity standard star HD122563 ( $V_{\text {hel }}=-26.51 \mathrm{~km} \mathrm{~s}^{-1}$; Chubak et al. 2012) obtained with the same instrument setup as the target stars. Depending on the $\mathrm{S} / \mathrm{N}$ of the target spectra, 30 to 50 individual orders were used for the cross-correlation. The mean value of the resulting velocities along with the standard deviation are listed in Table 1. Our values are different from the velocities determined for the stars by Simon et al. (2020), who find $V_{\text {hel }}=-108.6 \pm 1.0 \mathrm{~km} \mathrm{~s}^{-1}, \quad-108.1 \pm 1.0 \mathrm{~km} \mathrm{~s}^{-1}$, and $-109.3 \pm 1.0 \mathrm{~km} \mathrm{~s}^{-1}$ for $\mathrm{J} 220423, \mathrm{~J} 220409$, and $\mathrm{J} 220352$, respectively. However, J220423 and J220409 were both observed at high airmass, 1.8 and 1.6 respectively, which can cause offsets in the radial velocities ( $\mathrm{Ji}$ et al. 2020), and J220352 is likely in a binary system (Simon et al. 2020).

\section{Stellar Parameter Determination and Abundance Analysis}

Stellar parameter determination and abundance analysis was done following the techniques described in Hansen et al. (2017) and Marshall et al. (2019), using the 2017 version of MOOG (Sneden 1973) and making the assumption of local thermodynamic equilibrium (LTE) and including Rayleigh scattering treatment as described by Sobeck et al. (2011). ${ }^{52}$ The stellar parameters for the three stars were determined spectroscopically from equivalent width (EW) measurements of Fe I and Fe II lines. We measure the EW by fitting Gaussian profiles to the absorption lines in the continuum-normalized spectra. Uncertainties on the EWs were computed using $\sigma_{\mathrm{EW}}=$ $1.5 \sqrt{\mathrm{FWHM}} * \delta x / \mathrm{S} / \mathrm{N}$ from Cayrel (1988), where $\mathrm{S} / \mathrm{N}$ is the $\mathrm{S} / \mathrm{N}$ per pixel and $\delta x$ is the pixel size. First estimates of effective temperatures were determined from excitation equilibrium of Fe I lines. These were then placed on a photometric scale using the relation from Frebel et al. (2013). Using the corrected temperatures, surface gravities ( $\log g$ ) were determined from ionization equilibrium between the Fe I and Fe II lines. Finally, microturbulent velocities $(\xi)$ were determined by removing any trend in line abundances with reduced EW for the Fe I lines. Final stellar parameters along with estimated uncertainties are presented in Table 2 and lines used for the parameter determination of each star are listed in Table 3. All three stars are giants. For comparison, Table 2 also lists the photometric temperatures for our stars, which are in good agreement with the corrected spectroscopic temperatures. The photometric temperatures were derived by converting the $g, r, i$, and $z$ colors listed in Table 1 from DES DR1 (Abbott et al. 2018) to the corresponding $B, V, R$, and $I$ colors (DrlicaWagner et al. 2018), ${ }^{53}$ and using the $B-V, V-R, R-I$, and $V-I$ color temperature relations from Casagrande et al. (2010). Listed in Table 2 is the average photometric temperature and the standard deviation. Our derived metallicities are in good agreement with the metallicities derived from the Calcium Triplet lines by Simon et al. (2020), who find $[\mathrm{Fe} / \mathrm{H}]=-2.62 \pm 0.16,-2.72 \pm 0.16$, and $-2.93 \pm 0.22$ for J220423, J220409, and J220352, respectively.

Abundances were derived from EW measurements and spectral synthesis. EWs are used for strong nonblended lines

\footnotetext{
52 https://github.com/alexji/moog 17 scat

53 http://www.sdss3.org/dr8/algorithms/sdssUBVRITransform.php
} 
Table 1

Observing Log

\begin{tabular}{|c|c|c|c|c|c|c|c|c|c|c|c|c|}
\hline Object Name & $\begin{array}{l}\text { R.A. } \\
\text { (J2000) }\end{array}$ & $\begin{array}{l}\text { Decl. } \\
\text { (J2000) }\end{array}$ & $\begin{array}{c}g \\
(\mathrm{mag})\end{array}$ & $\begin{array}{c}r \\
(\mathrm{mag})\end{array}$ & $\begin{array}{c}i \\
(\mathrm{mag})\end{array}$ & $\begin{array}{c}z \\
(\mathrm{mag})\end{array}$ & $\begin{array}{l}\text { Date } \\
\text { (MJD) }\end{array}$ & $\begin{array}{l}t_{\exp } \\
(\mathrm{s})\end{array}$ & $\begin{array}{c}\mathrm{S} / \mathrm{N} \\
(\text { @ } 4100 \AA \text { A) }\end{array}$ & $\begin{array}{c}\mathrm{S} / \mathrm{N} \\
(@ 5500 \AA)\end{array}$ & $\begin{array}{c}V_{\text {hel }} \\
\left(\mathrm{km} \mathrm{s}^{-1}\right)\end{array}$ & Member \\
\hline DES J220423.91-463702.5 & 22:04:23.90 & $-46: 37: 02.48$ & 17.524 & 16.741 & 16.456 & 16.289 & 57605 & $7 \times 1800$ & 12 & 32 & $-106.9 \pm 0.6$ & Yes \\
\hline DES J220409.98-462102.2 & 22:04:09.98 & $-46: 21: 02.21$ & 18.157 & 17.477 & 17.227 & 17.086 & 57607 & $3 \times 3000$ & 9 & 17 & $-106.6 \pm 0.6$ & Yes \\
\hline DES J220352.01-462446.5 & 22:03:51.90 & $-46: 24: 46.40$ & 19.396 & 18.860 & 18.678 & 18.568 & $\begin{array}{l}57983 \\
58430\end{array}$ & $\begin{array}{c}2 \times 1800 \\
3 \times 1800+824\end{array}$ & $\begin{array}{l}9^{\mathrm{a}} \\
9^{\mathrm{a}}\end{array}$ & $\begin{array}{l}12^{\mathrm{a}} \\
12^{\mathrm{a}}\end{array}$ & $\begin{array}{l}-106.5 \pm 1.1 \\
-106.4 \pm 0.7\end{array}$ & Yes \\
\hline DES J220318.62-464116.4 & 22:03:18.62 & $-46: 41: 16.44$ & 18.726 & $\cdots$ & $\cdots$ & $\cdots$ & 58433 & $600+1800$ & 5 & 7 & $+76.3 \pm 0.7$ & No \\
\hline DES J220253.88-463522.6 & 22:02:53.88 & $-46: 35: 22.56$ & 18.703 & $\ldots$ & $\ldots$ & $\ldots$ & 58341 & 1800 & 3 & 5 & $+40.0 \pm 0.9$ & No \\
\hline
\end{tabular}

Note.

${ }^{\mathrm{a}}$ Signal-to-noise ratio $(\mathrm{S} / \mathrm{N})$ of the combined spectrum per pixel. 


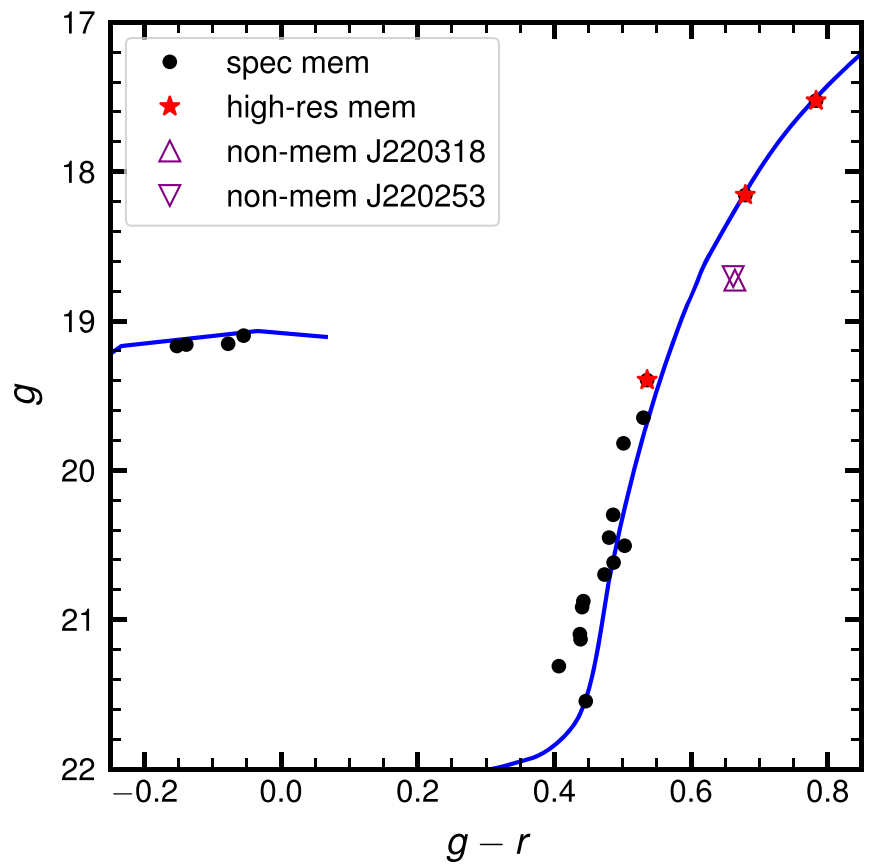

Figure 1. Color-magnitude diagram for Gru II. Black dots are confirmed spectroscopic members from Simon et al. (2020). Open triangle symbols are observed nonmembers; filled star symbols are observed members. Blue curves show a Dartmouth isochrone (Dotter et al. 2008) with $[\mathrm{Fe} / \mathrm{H}]=-2.2$ and age $=12.5 \mathrm{Gyr}$, and a M92 blue horizontal branch ridgeline from Belokurov et al. (2007) transformed to the DES photometric system and shifted to the distance of Gru II.

while spectral synthesis is used for weaker and or blended lines and for lines affected by isotopic and or hyperfine splitting. Measured EWs used for abundance determination are given in Table 4. All abundances were derived using $\alpha$-enhanced $([\alpha / \mathrm{Fe}]=+0.4) \quad$ 1D LTE ATLAS9 model atmospheres (Castelli \& Kurucz 2003) and the solar photosphere abundances from Asplund et al. (2009). As can be seen in Table 5, our stars are not enhanced in all $\alpha$ elements, hence using non$\alpha$-enhanced atmosphere models might be a better fit for our stars. However, Nagasawa et al. (2018) showed that for low $\mathrm{S} / \mathrm{N}$ spectra the difference in abundances was not more than 0.05 dex between using non- $\alpha$-enhanced and $\alpha$-enhanced atmosphere models to derive abundances for their low $\alpha$ stars of similar metallicity, which was much smaller than their total adopted uncertainty. We expect that the systematic differences in abundances caused by using $\alpha$-enhanced models in the work presented here to be similarly negligible compared to our abundance uncertainties. Hence, for more direct comparison with literature results we have used $\alpha$-enhanced atmosphere models. Line lists were generated using the linemake package ${ }^{54}$ (C. Sneden 2020, private communication), including molecular lines for $\mathrm{CH}, \mathrm{C}_{2}$, and $\mathrm{CN}$ and isotopic shift and hyperfine structure information. Representative errors arising from stellar parameter uncertainties computed for J220423 are listed in Table 6. These were determined by deriving abundances for the star using different atmospheric models, each with one parameter varied by its uncertainty as given in Table 2 . The uncertainties were then added in quadrature including covariance terms following McWilliam et al. (2013) and Johnson

\footnotetext{
54 https://github.com/vmplacco/linemake
}

(2002) to provide the systematic uncertainty, $\sigma_{\text {sys }}$, on $[\mathrm{X} / \mathrm{H}]$. The covariances were computed using the following equation

$$
\sigma_{X Y}=\frac{1}{N} \sum_{i}^{N}\left(X_{i}-\bar{X}\right)\left(Y_{i}-\bar{Y}\right) .
$$

To determine $\sigma_{T \log g}, \sigma_{T \xi}$, and $\sigma_{T[\mathrm{M} / \mathrm{H}]}, 20$ model atmospheres with effective temperatures drawn from a Gaussian distribution with a mean of $4585 \mathrm{~K}$ and standard deviation of $150 \mathrm{~K}$ were computed. $\log g$ and $\xi$ were then varied in turn until we obtained ionization equilibrium between the Fe I and Fe II lines for $\sigma_{T} \log g$, and no trend in line abundances with reduced $\mathrm{EW}$ of Fe I lines for $\sigma_{T \xi}$, while the direct change in $[\mathrm{Fe} / \mathrm{H}]$ was used for $\sigma_{T[\mathrm{M} / \mathrm{H}]}$. In the case of $\sigma_{\log g \xi}, 20$ model atmospheres with microturbulences drawn from a Gaussian distribution with a mean of $2.25 \mathrm{~km} \mathrm{~s}^{-1}$ and $\sigma$ of $0.3 \mathrm{~km} \mathrm{~s}^{-1}$ were computed and the gravity was then again varied to obtain ionization equilibrium between the $\mathrm{Fe}$ I and Fe II lines. The final covariances resulting from this process are $\sigma_{T \log g}=49$, $\sigma_{T \xi}=13, \sigma_{T[\mathrm{M} / \mathrm{H}]}=20$, and $\sigma_{\log g \xi}=-0.02$.

\section{Results}

Abundances or upper limits have been derived for 31 elements from $\mathrm{C}$ to $\mathrm{Er}$ in the three spectroscopically confirmed members of Gru II. All abundances and upper limits are presented in Table 5 listing the $\log _{\epsilon}(\mathrm{X}),[\mathrm{X} / \mathrm{H}]$, and $[\mathrm{X} / \mathrm{Fe}]$ abundances along with the number of lines used to derive the abundance, standard deviation $\left(\sigma_{\text {stat }}\right)$ and the total uncertainty on $[\mathrm{X} / \mathrm{H}]$ and $[\mathrm{X} / \mathrm{Fe}]$ calculated including covariance terms as described above. For elements where the abundances are derived from only one or two lines we use an estimated $\sigma_{[\mathrm{X} / \mathrm{H}] \text {,stat }}=0.2$ when calculating the total uncertainty. In Figure 3 we compare these abundances to results from high-resolution studies of other UFD galaxies and metal-poor MW halo stars (Roederer \& Kirby 2014). Only abundance detections have been included in the comparison sample. The UFD galaxies are: Boötes I (Feltzing et al. 2009; Norris et al. 2010; Gilmore et al. 2013; Ishigaki et al. 2014; Frebel et al. 2016), Boötes II (Ji et al. 2016b), Coma Berenices (Frebel et al. 2010), Grus I (Ji et al. 2019a), Hercules (Koch et al. 2008), Horologium I (Nagasawa et al. 2018), Leo IV (Simon et al. 2010), Pisces II (Spite et al. 2018), Reticulum II (Ji et al. 2016c), Segue 1 (Norris et al. 2010; Frebel et al. 2014), Segue 2 (Roederer et al. 2014), Triangulum II (Ji et al. 2019a), Tucana II (Ji et al. 2016d; Chiti et al. 2018), Tucana III (Hansen et al. 2017; Marshall et al. 2019), and Ursa Major II (Frebel et al. 2010).

\subsection{Carbon and Odd Z Elements}

Carbon abundances were determined from synthesis of the $\mathrm{CH} G$-band at $4313 \AA$ assuming a standard oxygen enhancement for metal-poor stars of $[\mathrm{O} / \mathrm{Fe}]=0.4$ as a direct oxygen measurement could not be made. $\mathrm{Na}$ abundances were derived from the 5889 and $5895 \AA$ lines via synthesis in J220409 and $\mathrm{J} 220423$, and EW analysis in $\mathrm{J} 220352$. Abundances for $\mathrm{Al}$ could only be determined for J220409 and J220423 and were determined via synthesis of the 3944 and $3961 \AA$ lines. The 7664 and $7698 \AA$ lines were used to derive the $\mathrm{K}$ abundances using EWs in J220423 and synthesis in J220409 and J220352. Finally a mixture of the Sc lines at 4226, 4320, 4400, 4415, 4670,5526 , and $5658 \AA$ was synthesized to derive Sc abundances in the stars. As can be seen in Figure 3 some 

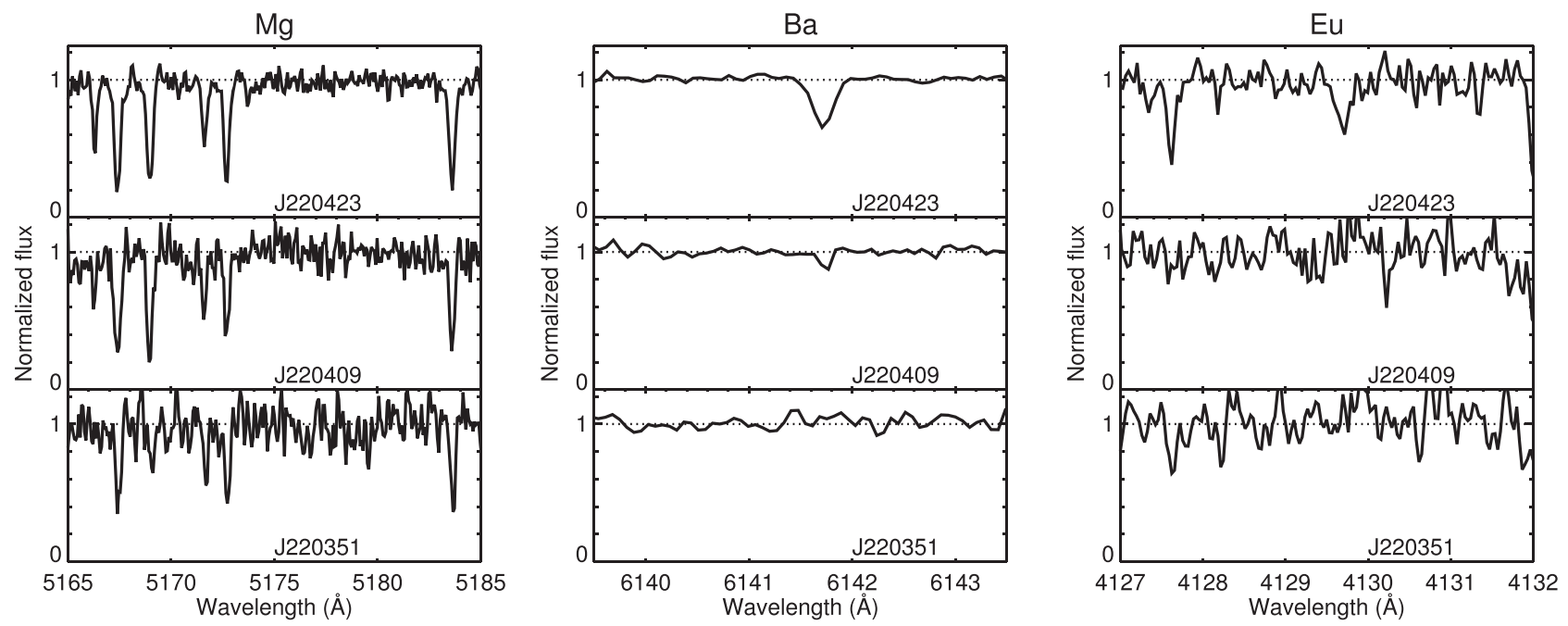

Figure 2. Spectra of the three stars around the Mg I b lines (left), the Ba II $6141 \AA$ A line (middle), and the Eu II $4129 \AA$ line (right).

Table 2

Measured Stellar Parameters

\begin{tabular}{lcccr}
\hline \hline ID & $\begin{array}{c}T_{\text {eff,photo }} \\
(\mathrm{K})\end{array}$ & $\begin{array}{c}T_{\text {eff,spec }} \text { ( } \\
(\mathrm{K})\end{array}$ & $\begin{array}{c}\log g \\
(\mathrm{cgs})\end{array}$ & $\begin{array}{c}\xi \\
\left(\mathrm{km} \mathrm{s}^{-1}\right)\end{array}$ \\
\hline DES J220423 & $4556 \pm 97$ & $4585 \pm 150$ & $1.22 \pm 0.3$ & $2.25 \pm 0.3$ \\
DES J220409 & $4740 \pm 73$ & $4720 \pm 150$ & $1.55 \pm 0.3$ & $2.35 \pm 0.3$ \\
DES J220352 & $5121 \pm 81$ & $4920 \pm 150$ & $1.91 \pm 0.3$ & $2.25 \pm 0.3$ \\
\hline
\end{tabular}

Note.

${ }^{\text {a }}$ Used to determine $\log g, \xi,[\mathrm{Fe} / \mathrm{H}]$, and for abundance analysis.

Table 3

EW and Atomic Data for Fe I and Fe II Lines used for Parameter Determination

\begin{tabular}{|c|c|c|c|c|c|c|c|}
\hline Stellar ID & Species & $\begin{array}{c}\lambda \\
(\AA)\end{array}$ & $\begin{array}{c}\chi \\
(\mathrm{eV})\end{array}$ & $\log g f$ & $\begin{array}{c}\text { EW } \\
(\mathrm{m} \AA)\end{array}$ & $\begin{array}{c}\sigma_{\mathrm{EW}} \\
(\mathrm{mA})\end{array}$ & $\log \epsilon$ \\
\hline J220409 & $\mathrm{Fe} \mathrm{I}$ & 3876.04 & 1.010 & -2.89 & 80.1 & 13.4 & 5.01 \\
\hline J220409 & $\mathrm{Fe} \mathrm{I}$ & 4001.66 & 2.174 & -1.90 & 51.4 & 13.3 & 4.91 \\
\hline J220409 & $\mathrm{Fe} \mathrm{I}$ & 4084.49 & 3.329 & -0.54 & 55.7 & 12.8 & 4.97 \\
\hline J220409 & $\mathrm{Fe} \mathrm{I}$ & 4132.90 & 2.840 & -0.92 & 41.6 & 10.7 & 4.54 \\
\hline J220409 & $\mathrm{Fe} \mathrm{I}$ & 4147.67 & 1.484 & -2.07 & 84.6 & 13.7 & 4.78 \\
\hline J220409 & $\mathrm{Fe} \mathrm{I}$ & 4156.80 & 2.830 & -0.81 & 46.2 & 11.5 & 4.49 \\
\hline$\vdots$ & $\vdots$ & $\vdots$ & $\vdots$ & $\vdots$ & $\vdots$ & $\vdots$ & \\
\hline J220409 & $\mathrm{Fe}$ II & 4508.29 & 2.860 & -2.25 & 48.2 & 13.9 & 4.67 \\
\hline J220409 & $\mathrm{Fe}$ II & 4515.34 & 2.840 & -2.36 & 52.3 & 14.7 & 4.81 \\
\hline J220409 & $\mathrm{Fe}$ II & 4520.22 & 2.810 & -2.60 & 41.6 & 13.9 & 4.85 \\
\hline J220409 & Fe II & 4522.63 & 2.840 & -1.99 & 93.7 & 18.6 & 5.12 \\
\hline
\end{tabular}

(This table is available in its entirety in machine-readable form.)

spread is detected in the $\mathrm{C}$ abundances for the three stars, however, effects of stellar evolution are known to alter the surface $\mathrm{C}$ abundance of low-mass stars. Following Placco et al. (2014) we find carbon corrections of $\Delta \mathrm{C}=+0.70$ for $\mathrm{J} 220423,+0.46$ for $\mathbf{J} 220409$, and +0.07 for $\mathbf{J} 220352$, reducing the spread in the birth carbon abundance of the stars. Taking these corrections into account, none of the stars qualify as carbon-enhanced metal-poor (CEMP) stars $([\mathrm{C} / \mathrm{Fe}]>0.7$; Aoki et al. 2007). In Figure 3 we plot the original C abundances for easy reference to the literature values. It should 
Table 4

EW Measurements and Atomic Data for Lines Used for Abundance Determination

\begin{tabular}{|c|c|c|c|c|c|c|c|}
\hline Stellar ID & Species & $\begin{array}{c}\lambda \\
(\stackrel{\bigcirc}{\AA})\end{array}$ & $\begin{array}{c}\chi \\
(\mathrm{eV})\end{array}$ & $\log g f$ & $\begin{array}{c}\mathrm{EW} \\
(\mathrm{m \AA})\end{array}$ & $\begin{array}{c}\sigma_{\mathrm{EW}} \\
(\mathrm{mA})\end{array}$ & $\log \epsilon$ \\
\hline J220409 & Mg I & 3986.75 & 4.343 & -1.44 & 21.7 & 9.7 & 5.38 \\
\hline J220409 & Mg I & 4057.51 & 4.343 & -1.20 & 40.1 & 10.4 & 5.49 \\
\hline J220409 & Mg I & 4167.27 & 4.343 & -1.00 & 50.2 & 12.7 & 5.44 \\
\hline J220409 & $\mathrm{Mg} \mathrm{I}$ & 4571.10 & 0.000 & -5.69 & 87.1 & 16.8 & 5.46 \\
\hline J220409 & $\mathrm{Mg} \mathrm{I}$ & 4702.99 & 4.343 & -0.67 & 75.4 & 15.0 & 5.41 \\
\hline J220409 & $\mathrm{Ca} \mathrm{I}$ & 4283.01 & 1.884 & -0.22 & 52.5 & 12.2 & 3.68 \\
\hline J220409 & $\mathrm{Ca} \mathrm{I}$ & 4318.65 & 1.897 & -0.21 & 62.0 & 13.3 & 3.83 \\
\hline J220409 & $\mathrm{Ca} \mathrm{I}$ & 4425.44 & 1.878 & -0.39 & 28.6 & 11.3 & 3.43 \\
\hline J220409 & $\mathrm{Ca} \mathrm{I}$ & 4455.89 & 1.897 & -0.51 & 27.2 & 10.9 & 3.54 \\
\hline J220409 & $\mathrm{Ca} \mathrm{I}$ & 5594.46 & 2.521 & -0.05 & 38.8 & 13.3 & 3.97 \\
\hline
\end{tabular}

(This table is available in its entirety in machine-readable form.)

be noted that many of the stars in the figure are giants, thus their original $\mathrm{C}$ abundance may be somewhat different from the measured $\mathrm{C}$ abundances plotted. For $\mathrm{Na}, \mathrm{Al}$, and $\mathrm{Sc}$ we find abundances of the three Gru II stars that are similar to other UFD and metal-poor halo stars (see Figure 3). K abundances have only been derived for stars in a few UFD galaxies but the majority of these seem to cluster around $[\mathrm{K} / \mathrm{Fe}]=0.7$ while the stars in Gru II exhibit lower $\mathrm{K}$ abundances around $[\mathrm{K} / \mathrm{Fe}]=0.3$, closer to the abundances found in the halo comparison sample (Roederer \& Kirby 2014).

\section{2. $\alpha$ and Iron-peak Elements}

EW analysis was used to derive $\mathrm{Mg}$ and $\mathrm{Ti}$ abundances in all three stars. Ca abundances were also derived from EW analysis in J220409 and J220423 while spectral synthesis of the Ca lines at 4426, 4434, 4454, 6122, and $6162 \AA$ was used to determine the $\mathrm{Ca}$ abundance in $\mathrm{J} 220352$. $\mathrm{Cr}$ and $\mathrm{Ni}$ abundances were determined using EW analysis in J220423, while synthesis of the $\mathrm{Cr}$ lines at 4252, 4274, 4289, 4646, and $5206 \AA$ and Ni lines at 3807,5476 , and $6644 \AA$ was used in J220409 and J220352. For the remaining elements in this group, $\mathrm{Si}, \mathrm{V}, \mathrm{Mn}, \mathrm{Co}, \mathrm{Cu}$, and $\mathrm{Zn}$, abundances or upper limits were determined via spectral synthesis in all three stars using

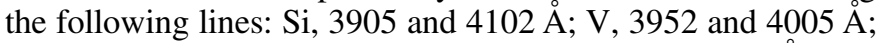
Mn, 4030, 4033, 4034, 4041, 4754, 4823, and $4762 \AA$; Co, 4118 and $4121 \AA$; $\mathrm{Cu}, 5105 \AA$; and $\mathrm{Zn}, 4722$ and $4810 \AA$. All three stars are enhanced in $\mathrm{Mg}$, similar to other metal-poor stars in the halo and UFDs. However the derived abundances for $\mathrm{Si}$, $\mathrm{Ca}$, and $\mathrm{Ti}$, which are also usually enhanced in metal-poor stars, are low in all three Gru II stars compared to the halo and UFD galaxy sample. $\mathrm{Mg}, \mathrm{Si}, \mathrm{Ca}$, and Ti are primarily created in massive $\left(M>8 M_{\odot}\right)$ stars, with $\mathrm{Si}, \mathrm{Ca}$, and $\mathrm{Ti}$ being synthesized during the explosive nucleosynthesis in the CCSN phase and $\mathrm{Mg}$ being created during the hydrostatic burning phases of the stars (Woosley \& Weaver 1995). A high ratio of hydrostatic to explosive $\alpha$-elements as observed in these stars can be produced in high mass, $\geqslant 20 M_{\odot}$ CCSN (Heger \& Woosley 2010). The three Gru II stars also exhibit low $\mathrm{Cr}$ abundances, a feature in common with stars in other UFD galaxies and also found for the most metal-poor MW halo stars (McWilliam et al. 1995). The abundances derived for V, Mn, $\mathrm{Co}, \mathrm{Ni}$, and $\mathrm{Zn}$ in the Gru II stars follow the abundance trends observed in other UFDs and metal-poor halo stars (see Figure 3).

\subsection{Neutron-capture Elements}

Abundances for all neutron-capture elements were derived from spectral synthesis. It was only possible to derive abundances for $\mathrm{Sr}$ in all three stars using the 4077 and $4215 \AA$ lines. $\mathrm{Ba}$ abundances for J220904 and J220423 and the upper limit in J220352 were derived from the 5853, 6141, and $6496 \AA$ lines. The most metal-rich of the three stars J220423 exhibits a small enhancement in some of the heavy neutron-capture elements $([\mathrm{Eu} / \mathrm{Fe}]=0.31 \pm 0.22)$, enabling the derivation of abundances for Y (4398 $\AA$ ), Zr (4149 ̊), La (3995 and $4086 \AA)$, Pr (4179, 4222, and 4408 $)$, Nd (4109, 4177, 4462, and $4825 \AA$ ), and $\mathrm{Eu}(3907,4129$, and $4205 \AA$ ) for this star. Eu upper limits derived from the $4129 \AA$ line are given for J220904 and J220352 also. The $\mathrm{Sr}$ and $\mathrm{Ba}$ abundances derived for $\mathrm{J} 220423$ are subsolar but higher than what is found in the other two stars analyzed, suggesting that an additional source of neutron-capture elements has enriched this star. $\mathbf{J} 220423$ has $[\mathrm{Ba} / \mathrm{Eu}]=-0.80$, compatible with an $r$-process origin of the neutron-capture element excess (Sneden et al. 2008). The two nonenhanced stars J220409 and $\mathrm{J} 220352$ both exhibit extremely low $\mathrm{Sr}$ (and $\mathrm{Ba}$ ) abundances similar to neutron-capture element abundances detected in other UFDs (Ji et al. 2019a), supporting the dwarf galaxy classification of Gru II (see Figure 3).

\section{Discussion}

For the majority of the elements, the abundances derived for Gru II follow the trends detected in other UFD galaxies and in the MW halo, with a few notable outliers. In Figure 4 we plot the $[\mathrm{Mg} / \mathrm{Ca}]$ ratio as a function of metallicity for the three Gru II stars along with the UFD galaxies and halo stars from Figure 3. The three stars in Gru II clearly stand out, displaying somewhat higher $[\mathrm{Mg} / \mathrm{Ca}]$ ratios than the majority of the comparison sample stars. This $\alpha$-element signature was first discovered in the MW halo star CS 22876-037 (black square in Figure 4) for which Norris et al. (2000) reported abundances of $[\mathrm{Mg} / \mathrm{Fe}]=0.5 \pm 0.12$ and $[\mathrm{Ca} / \mathrm{Fe}]=0.01 \pm 0.13$. A more extreme version of this abundance signature was later observed in another MW halo star, namely HE 1424-0241 (black diamond in Figure 4), where Cohen et al. (2007) reported abundances of $[\mathrm{Mg} / \mathrm{Fe}]=0.44 \pm 0.12$ and $[\mathrm{Ca} / \mathrm{Fe}]=$ $-0.58 .^{55}$ Both stars display a high dominance of hydrostatic

\footnotetext{
$\overline{55}$ No uncertainty was reported on the $\mathrm{Ca}$ abundance as it was determined from just one absorption feature.
} 
Table 5

Abundances and Associated Uncertainties Derived for the Three Stars

\begin{tabular}{|c|c|c|c|c|c|c|c|c|c|c|c|c|c|c|c|c|c|c|c|c|c|}
\hline \multirow[b]{2}{*}{ Element } & \multicolumn{7}{|c|}{$\mathrm{J} 220409$} & \multicolumn{7}{|c|}{$\mathrm{J} 220423$} & \multicolumn{7}{|c|}{ J220351 } \\
\hline & $\begin{array}{c}\log \epsilon(\mathrm{X}) \\
(\operatorname{dex})\end{array}$ & $N$ & $\begin{array}{c}\sigma_{\text {stat }} \\
(\mathrm{dex})\end{array}$ & $\begin{array}{c}{[\mathrm{X} / \mathrm{H}]} \\
(\mathrm{dex})\end{array}$ & $\begin{array}{l}\sigma_{[\mathrm{X} / \mathrm{H}]} \\
(\mathrm{dex})\end{array}$ & $\begin{array}{c}{[\mathrm{X} / \mathrm{Fe}]} \\
(\mathrm{dex})\end{array}$ & $\begin{array}{c}\sigma_{[\mathrm{X} / \mathrm{Fe}]} \\
(\mathrm{dex})\end{array}$ & $\begin{array}{c}\log \epsilon(\mathrm{X}) \\
(\operatorname{dex})\end{array}$ & $N$ & $\begin{array}{c}\sigma_{\text {stat }} \\
(\operatorname{dex})\end{array}$ & $\begin{array}{c}{[\mathrm{X} / \mathrm{H}]} \\
(\mathrm{dex})\end{array}$ & $\begin{array}{c}\sigma_{[\mathrm{X} / \mathrm{H}]} \\
(\mathrm{dex})\end{array}$ & $\begin{array}{c}{[\mathrm{X} / \mathrm{Fe}]} \\
(\mathrm{dex})\end{array}$ & $\begin{array}{c}\sigma_{[\mathrm{X} / \mathrm{Fe}]} \\
(\mathrm{dex})\end{array}$ & $\begin{array}{c}\log \epsilon(\mathrm{X}) \\
(\operatorname{dex})\end{array}$ & $N$ & $\begin{array}{c}\sigma_{\text {stat }} \\
(\mathrm{dex})\end{array}$ & $\begin{array}{c}{[\mathrm{X} / \mathrm{H}]} \\
(\mathrm{dex})\end{array}$ & $\begin{array}{c}\sigma_{[\mathrm{X} / \mathrm{H}]} \\
(\mathrm{dex})\end{array}$ & $\begin{array}{c}{[\mathrm{X} / \mathrm{Fe}]} \\
(\mathrm{dex})\end{array}$ & $\begin{array}{c}\sigma_{[\mathrm{X} / \mathrm{Fe}]} \\
(\mathrm{dex})\end{array}$ \\
\hline C & 5.41 & & 0.20 & -3.02 & 0.47 & -0.33 & 0.31 & 5.22 & & 0.20 & -3.21 & 0.47 & -0.72 & 0.32 & 6.01 & & 0.20 & -2.44 & 0.47 & 0.52 & 0.30 \\
\hline $\mathrm{N}$ & $<+7.83$ & & $\ldots$ & $<-0.69$ & $\ldots$ & $<+2.00$ & $\ldots$ & $<+7.34$ & & $\cdots$ & $<-0.49$ & $\ldots$ & $<+2.00$ & $\ldots$ & $<+6.89$ & & $\ldots$ & $<-0.94$ & $\ldots$ & $<+2.00$ & $\cdots$ \\
\hline $\mathrm{Na} I$ & 3.57 & 2 & 0.20 & -2.67 & 0.30 & 0.02 & 0.27 & 3.85 & 2 & 0.20 & -2.39 & 0.30 & 0.10 & 0.28 & 2.85 & 2 & 0.32 & -3.39 & 0.39 & -0.45 & 0.36 \\
\hline Mg I & 5.44 & 5 & 0.17 & -2.18 & 0.27 & 0.51 & 0.25 & 5.55 & 5 & 0.21 & -2.05 & 0.30 & 0.44 & 0.29 & 5.38 & 2 & 0.20 & -2.22 & 0.29 & 0.72 & 0.26 \\
\hline $\mathrm{Al} \mathrm{I}$ & 2.79 & 2 & 0.20 & -3.66 & 0.50 & -0.97 & 0.32 & 3.15 & 2 & 0.20 & -3.30 & 0.46 & -0.81 & 0.26 & $\ldots$ & & $\ldots$ & $\ldots$ & $\ldots$ & $\ldots$ & $\ldots$ \\
\hline Si I & 4.89 & 1 & 0.20 & -2.62 & 0.43 & 0.07 & 0.28 & 5.22 & 1 & 0.20 & -2.29 & 0.43 & 0.20 & 0.38 & 4.62 & 2 & 0.20 & -2.89 & 0.43 & 0.05 & 0.26 \\
\hline K I & 2.55 & 2 & 0.20 & -2.48 & 0.30 & 0.21 & 0.27 & 2.96 & 2 & 0.20 & -2.07 & 0.30 & 0.42 & 0.28 & 2.34 & 1 & 0.20 & -2.69 & 0.30 & 0.25 & 0.26 \\
\hline $\mathrm{Ca} I$ & 3.69 & 13 & 0.26 & -2.65 & 0.32 & 0.04 & 0.33 & 3.96 & 13 & 0.24 & -2.38 & 0.30 & 0.11 & 0.32 & 3.55 & 5 & 0.27 & -2.79 & 0.33 & 0.15 & 0.33 \\
\hline ScII & 0.55 & 6 & 0.09 & -2.60 & 0.24 & 0.09 & 0.23 & 0.44 & 5 & 0.16 & -2.71 & 0.27 & -0.22 & 0.28 & 0.27 & 2 & 0.20 & -2.88 & 0.30 & 0.06 & 0.28 \\
\hline Ti I & 2.33 & 4 & 0.23 & -2.62 & 0.38 & 0.07 & 0.29 & 2.48 & 8 & 0.20 & -2.47 & 0.36 & 0.02 & 0.27 & $\cdots$ & & $\ldots$ & $\ldots$ & $\cdots$ & $\ldots$ & $\ldots$ \\
\hline TiII & 2.34 & 11 & 0.25 & -2.61 & 0.29 & 0.08 & 0.33 & 2.58 & 19 & 0.22 & -2.37 & 0.26 & 0.12 & 0.32 & 1.79 & 7 & 0.29 & -3.16 & 0.32 & -0.22 & 0.36 \\
\hline VII & 1.52 & 2 & 0.20 & -2.41 & 0.26 & 0.28 & 0.30 & 1.50 & 1 & 0.20 & -2.43 & 0.26 & 0.06 & 0.31 & $<+1.73$ & & $\ldots$ & $<-2.44$ & $\ldots$ & $<+0.50$ & $\ldots$ \\
\hline Cr I & 2.53 & 4 & 0.10 & -3.11 & 0.27 & -0.42 & 0.21 & 2.62 & 7 & 0.22 & -3.02 & 0.34 & -0.53 & 0.29 & 2.06 & 2 & 0.23 & -3.58 & 0.34 & -0.64 & 0.28 \\
\hline Mn I & 2.22 & 3 & 0.30 & -3.21 & 0.56 & -0.52 & 0.39 & 2.43 & 7 & 0.17 & -3.00 & 0.50 & -0.51 & 0.31 & 2.29 & 1 & 0.20 & -3.14 & 0.52 & -0.20 & 0.31 \\
\hline Fe I & 4.82 & 94 & 0.17 & -2.69 & 0.34 & $\ldots$ & $\ldots$ & 5.01 & 116 & 0.18 & -2.49 & 0.34 & $\ldots$ & $\ldots$ & 4.57 & 32 & 0.15 & -2.94 & 0.33 & $\ldots$ & $\ldots$ \\
\hline Fe II & 4.81 & 10 & 0.21 & -2.76 & 0.25 & $\ldots$ & $\ldots$ & 5.01 & 14 & 0.16 & -2.49 & 0.20 & $\ldots$ & $\ldots$ & 4.56 & 10 & 0.25 & -2.95 & 0.28 & $\ldots$ & $\ldots$ \\
\hline Co I & 2.25 & 1 & 0.20 & -2.74 & 0.39 & -0.05 & 0.27 & 2.31 & 2 & 0.20 & -2.68 & 0.39 & -0.19 & 0.28 & 1.92 & 1 & 0.20 & -3.07 & 0.39 & -0.13 & 0.26 \\
\hline $\mathrm{Ni} \mathrm{I}$ & 3.29 & 2 & 0.20 & -2.93 & 0.21 & -0.24 & 0.29 & 3.70 & 7 & 0.26 & -2.52 & 0.32 & -0.03 & 0.34 & $\ldots$ & & $\ldots$ & $\ldots$ & $\ldots$ & $\ldots$ & $\ldots$ \\
\hline $\mathrm{Cu} \mathrm{I}$ & $<+1.40$ & & $\ldots$ & $<-2.79$ & $\ldots$ & $<-0.10$ & $\ldots$ & $<+1.40$ & & $\ldots$ & $<-2.79$ & $\ldots$ & $<-0.30$ & $\ldots$ & $<+2.05$ & & $\cdots$ & $<-2.14$ & $\cdots$ & $<+0.80$ & $\cdots$ \\
\hline $\mathrm{Zn} \mathrm{I}$ & $<+2.07$ & & $\ldots$ & $<-2.49$ & $\ldots$ & $<+0.20$ & $\ldots$ & 2.07 & 2 & 0.20 & -2.49 & 0.29 & 0.00 & 0.30 & $<+2.32$ & & $\cdots$ & $<-2.24$ & $\ldots$ & $<+0.70$ & $\cdots$ \\
\hline Sr II & -1.70 & 2 & 0.20 & -4.57 & 0.29 & -1.88 & 0.31 & -0.75 & 2 & 0.20 & -3.62 & 0.29 & -1.13 & 0.32 & -1.97 & 1 & 0.20 & -4.84 & 0.29 & -1.90 & 0.31 \\
\hline Y II & $<-0.78$ & & $\cdots$ & $<-2.99$ & $\ldots$ & $<-0.30$ & $\ldots$ & -1.18 & 1 & 0.20 & -3.39 & 0.23 & -0.90 & 0.33 & $<-0.73$ & & $\ldots$ & $<-2.94$ & $\ldots$ & $<+0.00$ & $\cdots$ \\
\hline Zr II & $<-0.61$ & & $\cdots$ & $<-3.19$ & $\cdots$ & $<-0.50$ & $\cdots$ & -0.41 & 1 & 0.20 & -2.99 & 0.30 & -0.50 & 0.29 & $<-0.36$ & & $\cdots$ & $<-2.94$ & $\cdots$ & $<+0.00$ & $\cdots$ \\
\hline Ba II & -1.77 & 3 & 0.19 & -3.95 & 0.38 & -1.26 & 0.26 & -0.80 & 3 & 0.06 & -2.98 & 0.33 & -0.49 & 0.19 & $<-2.26$ & & $\ldots$ & $<-4.44$ & $\ldots$ & $<-1.50$ & $\ldots$ \\
\hline La II & $<-1.59$ & & $\cdots$ & $<-2.69$ & $\cdots$ & $<+0.00$ & $\cdots$ & -1.21 & 2 & 0.20 & -2.31 & 0.53 & 0.18 & 0.33 & $<-1.04$ & & $\cdots$ & $<-2.14$ & $\cdots$ & $<+0.80$ & $\cdots$ \\
\hline Ce II & $<-0.91$ & & $\cdots$ & $<-2.49$ & $\cdots$ & $<+0.20$ & $\cdots$ & $<-0.61$ & & $\cdots$ & $<-2.19$ & $\cdots$ & $<+0.30$ & $\cdots$ & $<-0.56$ & & $\cdots$ & $<-2.14$ & $\cdots$ & $<+0.80$ & $\cdots$ \\
\hline Pr II & $<-1.17$ & & $\cdots$ & $<-1.89$ & $\cdots$ & $<+0.80$ & $\cdots$ & -1.48 & 3 & 0.03 & -2.20 & 0.26 & 0.29 & 0.20 & $\cdots$ & & $\cdots$ & $\cdots$ & $\cdots$ & $\cdots$ & $\cdots$ \\
\hline Nd II & $<-0.57$ & & $\ldots$ & $<-1.99$ & $\ldots$ & $<+0.70$ & $\ldots$ & -0.99 & 4 & 0.07 & -2.41 & 0.24 & 0.08 & 0.23 & $<-0.58$ & & $\ldots$ & $<-2.04$ & $\ldots$ & $<+0.90$ & $\ldots$ \\
\hline Sm II & $\ldots$ & & $\ldots$ & $\ldots$ & $\ldots$ & $\ldots$ & $\ldots$ & $<-1.03$ & & $\ldots$ & $<-1.99$ & $\ldots$ & $<+0.50$ & $\ldots$ & $<-0.88$ & & $\ldots$ & $<-1.84$ & $\ldots$ & $<+1.10$ & $\ldots$ \\
\hline Eu II & $<-2.07$ & & $\ldots$ & $<-2.59$ & $\ldots$ & $<+0.10$ & $\ldots$ & -1.66 & 3 & 0.10 & -2.18 & 0.21 & 0.31 & 0.25 & $<-1.92$ & & $\cdots$ & $<-1.44$ & $\cdots$ & $<+0.50$ & $\cdots$ \\
\hline Gd II & $<-0.92$ & & $\ldots$ & $<-1.99$ & $\ldots$ & $<+0.70$ & $\ldots$ & $<-0.62$ & & $\ldots$ & $<-1.69$ & $\ldots$ & $<+0.80$ & $\ldots$ & $\ldots$ & & $\ldots$ & $\ldots$ & $\ldots$ & $\ldots$ & $\cdots$ \\
\hline Dy II & $<-0.59$ & & $\ldots$ & $<-1.69$ & $\ldots$ & $<+1.00$ & $\ldots$ & $<-0.69$ & & $\ldots$ & $<-1.79$ & $\ldots$ & $<+0.70$ & $\cdots$ & $\ldots$ & & $\cdots$ & $\ldots$ & $\cdots$ & $\ldots$ & $\ldots$ \\
\hline Er II & $<-1.27$ & & $\ldots$ & $<-1.19$ & $\ldots$ & $<+0.50$ & $\ldots$ & $<-1.07$ & & $\ldots$ & $<-1.99$ & $\ldots$ & $<+0.50$ & $\ldots$ & $\ldots$ & & $\ldots$ & $\cdots$ & $\ldots$ & $\ldots$ & $\ldots$ \\
\hline
\end{tabular}


Table 6

Abundance Errors Arising from Stellar Parameter Uncertainties for DES J220423

\begin{tabular}{lccccc}
\hline \hline Element & $\begin{array}{c}\Delta T_{\text {eff }} \\
(\text { dex })\end{array}$ & $\begin{array}{c}\Delta \log g \\
(\text { dex })\end{array}$ & $\begin{array}{c}\Delta V_{\text {mic }} \\
(\text { dex })\end{array}$ & $\begin{array}{c}\Delta[\mathrm{M} / \mathrm{H}] \\
(\text { dex })\end{array}$ & $\begin{array}{c}\sigma_{\text {sys }} \\
(\text { dex })\end{array}$ \\
\hline CH & 0.27 & 0.12 & 0.04 & 0.04 & 0.43 \\
Na I & 0.15 & 0.03 & 0.10 & 0.00 & 0.22 \\
Mg I & 0.14 & 0.04 & 0.07 & 0.01 & 0.21 \\
$\mathrm{Al} \mathrm{I}$ & 0.22 & 0.13 & 0.07 & 0.14 & 0.45 \\
Si I & 0.25 & 0.05 & 0.15 & 0.02 & 0.38 \\
K I & 0.17 & 0.02 & 0.06 & 0.01 & 0.22 \\
Ca I & 0.13 & 0.03 & 0.05 & 0.01 & 0.18 \\
Sc I & 0.08 & 0.04 & 0.17 & 0.00 & 0.22 \\
Ti I & 0.25 & 0.03 & 0.05 & 0.01 & 0.30 \\
Ti II & 0.04 & 0.09 & 0.06 & 0.01 & 0.14 \\
V II & 0.05 & 0.10 & 0.02 & 0.02 & 0.16 \\
Cr I & 0.22 & 0.02 & 0.02 & 0.01 & 0.25 \\
Mn I & 0.30 & 0.05 & 0.10 & 0.12 & 0.47 \\
Fe I & 0.21 & 0.03 & 0.10 & 0.02 & 0.29 \\
Fe II & 0.02 & 0.10 & 0.05 & 0.01 & 0.13 \\
Co I & 0.25 & 0.03 & 0.07 & 0.04 & 0.33 \\
Ni I & 0.16 & 0.01 & 0.02 & 0.01 & 0.18 \\
Zn I & 0.10 & 0.10 & 0.00 & 0.00 & 0.21 \\
Sr II & 0.02 & 0.02 & 0.20 & 0.05 & 0.21 \\
Y II & 0.07 & 0.03 & 0.03 & 0.00 & 0.11 \\
Zr II & 0.09 & 0.13 & 0.04 & 0.00 & 0.23 \\
Ba II & 0.13 & 0.16 & 0.08 & 0.05 & 0.33 \\
La II & 0.20 & 0.22 & 0.11 & 0.10 & 0.49 \\
Pr II & 0.16 & 0.07 & 0.03 & 0.04 & 0.26 \\
Nd II & 0.12 & 0.08 & 0.00 & 0.04 & 0.22 \\
Eu II & 0.09 & 0.08 & 0.01 & 0.02 & 0.18 \\
\hline & & & & & \\
\hline
\end{tabular}

versus explosive $\alpha$-elements. From the CCSNe models of Woosley \& Weaver (1995), Norris et al. (2000) conclude that CS 22876-037 was likely enriched by a zero metallicity $30 M_{\odot}$ CCSN. Using the online fitting code STARFIT ${ }^{56}$ which performs a $\chi^{2}$ fit of $\mathrm{SNe}$ models to abundances data (see Heger \& Woosley 2010, and subsequent online update in 2012 for more details), we fit $\mathrm{SN}$ models to the $\mathrm{C}, \mathrm{Na}, \mathrm{Mg}, \mathrm{Ca}, \mathrm{Ti}$, $\mathrm{Fe}, \mathrm{Co}$, and $\mathrm{Ni}$ abundances of the three Gru II stars. Carbon values corrected for stellar evolution were used for the fit. Abundances of Sc and $\mathrm{Cr}$ have been excluded as these elements are generally underproduced in these models (Heger \& Woosley 2010). The result is shown in Figure 5 where dashed lines represent the best fit using all elements and solid lines represent fits using only the $\mathrm{Mg}, \mathrm{Ca}$, and $\mathrm{Fe}$ abundances, demonstrating that higher-mass models are preferred when only considering the $\mathrm{Mg}, \mathrm{Ca}$, and $\mathrm{Fe}$ abundances detected in these stars. This agrees with the results from Norris et al. (2000), which suggest that stars with this chemical signature were likely enriched by a population of very high-mass stars. It is notable that in Figure 4, stars in two other systems also stand out, namely Her (blue points) and Psc II (orange-red point). The high $[\mathrm{Mg} / \mathrm{Ca}]$ in the star in Psc II is driven by a very high $\mathrm{Mg}$ abundance (see Figure 3). Furthermore, this star also exhibits a high $\mathrm{C}$ abundance and is classified as a CEMP-no star (Spite et al. 2018). The source of carbon in CEMP-no stars is still debated, but rotation and mixing in the progenitor stars is likely to play a significant role. This has also been shown to influence the production of other light elements such as $\mathrm{Mg}$ (Maeder et al. 2015). Hence it is not clear if $[\mathrm{Mg} / \mathrm{Ca}]$ ratios in

\footnotetext{
56 http://starfit.org/
}

CEMP-no and non-carbon enhanced stars, like the Gru II stars, should be discussed in the same context. In Her, an $\alpha$-element abundance signature similar to that detected in Gru II is seen in both stars analyzed by Koch et al. (2008), who found $[\mathrm{Mg} / \mathrm{Ca}]=0.94$ and 0.58 for the two stars. Hence, like the stars in Gru II, the stars in Her were also likely enriched by a population of high-mass stars. Koch et al. (2008) suggest that this is either the result of an IMF of Her skewed toward highermass stars or stochastic chemical evolution in this galaxy. In the following, we discuss these two and alternative scenarios to explain the abundance signature of Gru II.

\subsection{Top-heavy IMF}

Some correlations have been detected between IMFs and galaxy properties, with the largest galaxies having bottomheavy IMFs and smaller galaxies having more bottom-light IMFs (Geha et al. 2013, although Gennaro et al. 2018 find a more complicated picture). As previously described, UFDs are ideal laboratories for studying the low-mass stellar IMF. Geha et al. (2013) determined the IMF for Her based on Hubble Space Telescope imaging and detected a slope of $\alpha=1.2$. This is a somewhat shallower slope than the Salpeter $(\alpha=2.35$; Salpeter 1955) or Kroupa ( $\alpha=2.3$; Kroupa 2001) IMF detected for more massive systems. On the other hand, this result is in good agreement with the high $[\mathrm{Mg} / \mathrm{Ca}]$ abundance signature of the galaxy, and hence an overall chemical enrichment dominated by high-mass stars. Again, it should be noted that the result of Geha et al. (2013) is based on the low-mass $\left(M<0.8 M_{\odot}\right)$ stellar population observable in Her today and does not necessarily directly translate to the IMF of higher-mass stellar populations previously present in the galaxy. With only three stars analyzed in Gru II it is difficult to conclude if the overall chemical enrichment of the galaxy is dominated by high-mass stars. However, the very consistent $\alpha$-element signature detected in these three stars, requiring nucleosynthesis in $\geqslant 20 M_{\odot}$ stars to produce, is compatible with a top-heavy IMF for the Population III stars, leading to a different chemical signature in this galaxy than that seen in other UFD galaxies. It should be noted that Geha et al. (2013) also measured the IMF for Leo IV and found a slope of $\alpha=1.3$ similar to Her. Chemical abundances from highresolution data have only been presented for one star in Leo IV (Simon et al. 2010). This star shows roughly equal, small $\mathrm{Mg}$ and $\mathrm{Ca}$ enhancements with $[\mathrm{Mg} / \mathrm{Ca}]=0.07$, thus not similar to the $\alpha$-elements signature found in Her and Gru II.

\subsection{Stochastic Chemical Enrichment}

Another possible explanation for the chemically peculiar stars in Gru II is that the chemical enrichment and star formation in systems like Gru II are stochastic and inhomogeneous. It can be seen in Figure 4 that other UFD galaxy stars exhibit a similar $\alpha$-element signature as the three Gru II and two Her stars, including the $r$-process enhanced galaxies Ret II and Tuc III ( Ji et al. 2016c; Marshall et al. 2019). In these systems, however, the high $[\mathrm{Mg} / \mathrm{Ca}]$ stars are outliers and the majority of the stars exhibit enhancements in the $\alpha$ elements similar to metal-poor halo stars. Since the study of Koch et al. (2008), François et al. (2016) have derived $\mathrm{Mg}$ and $\mathrm{Ca}$ abundances for an additional four stars in Her from medium-resolution spectra (data not included in Figures 3 and 4). The stars in this sample are more metal-poor $(-2.83<[\mathrm{Fe} / \mathrm{H}]<-2.28)$ and only one of the 

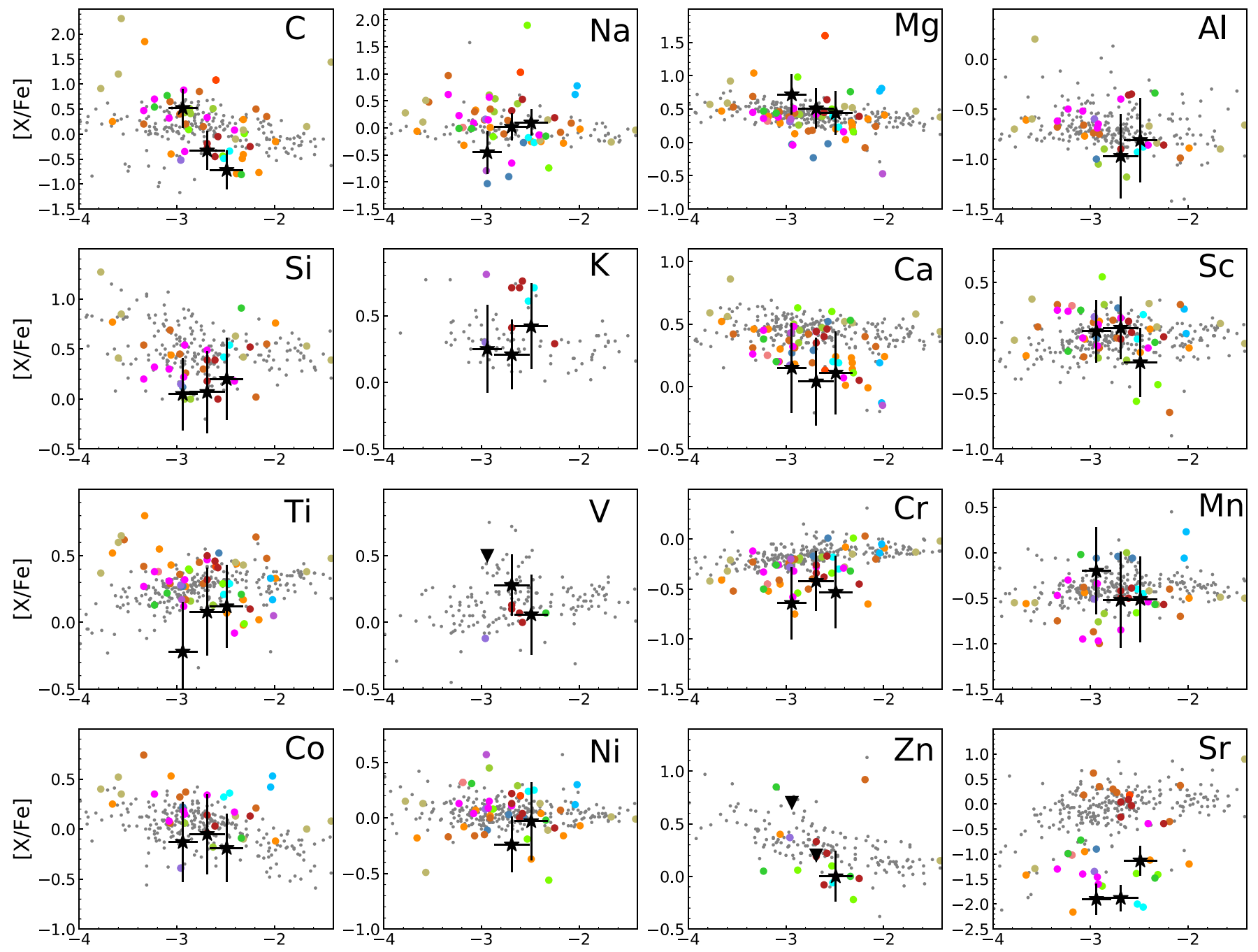

$[\mathrm{Fe} / \mathrm{H}]$
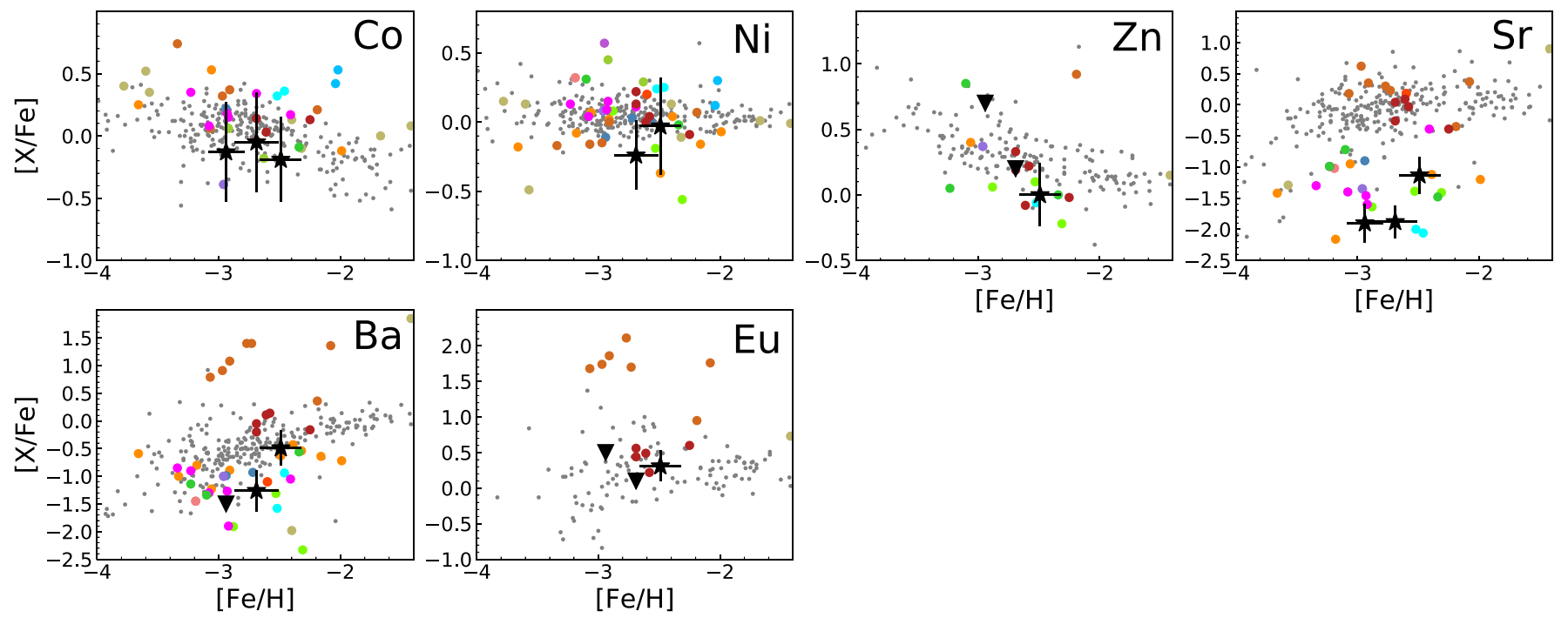

$[\mathrm{Fe} / \mathrm{H}]$

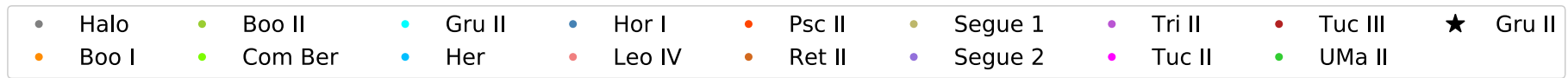

Figure 3. $[\mathrm{X} / \mathrm{Fe}]$ derived abundances for Gru II (black stars) compared to stellar abundances from the MW halo (gray dots; Roederer \& Kirby 2014) and other UFD galaxies (colored dots according to legend, see text for references). Upper limits for Gru II stars are marked with downward pointing black triangles.

four stars show an $\alpha$-elements signature $([\mathrm{Mg} / \mathrm{Ca}]=0.24)$ similar to the Koch et al. (2008) stars. Including these stars, the chemical signature of Her more resembles that of the other UFD galaxies, suggesting that stochastic and inhomogeneous chemical enrichment of Her is more likely the explanation for the varying abundance patterns detected in Her. For the abundance signature to be the result of stochastic chemical enrichment of a given galaxy, only a few chemical enrichment events can have polluted the galaxy overall. The more enrichment events a system encounters, the more washed-out the signature of any individual event becomes. Koch et al. (2008) performed a stochastic sampling following McWilliam \& Searle (1999) and found that the abundance ratios of their two Her stars could only reasonably be obtained in a system with fewer than $11 \mathrm{SNe}$. They note that this is somewhat at odds with the iron abundances of their stars of $[\mathrm{Fe} / \mathrm{H}] \sim-2$, which likely requires on the order of $100 \mathrm{SNe}$ to obtain (Koch et al. 2008). The halo stars exhibiting the $\alpha$-element signature are found at very-low metallicity $([\mathrm{Fe} / \mathrm{H}]<-3.5)$, making it more likely that the gas from which these formed was enriched by just one SN. The three Gru II stars have metallicities between $-2.94<[\mathrm{Fe} / \mathrm{H}]<-2.49$, thus it is not unlikely that the gas from which these stars formed was enriched by a low 


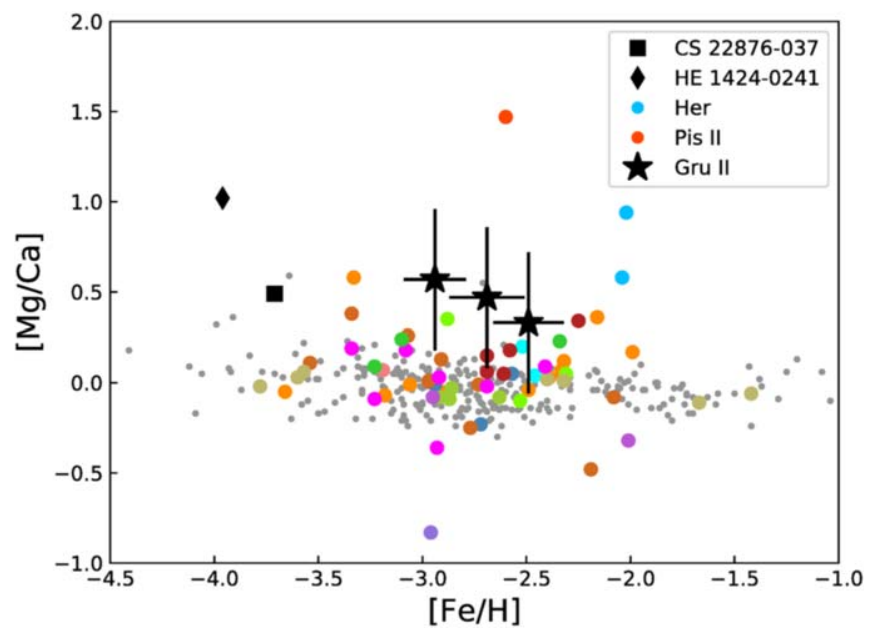

Figure 4. $[\mathrm{Mg} / \mathrm{Ca}]$ as a function of metallicity for the three Gru II stars compared to stars in other UFDs and metal-poor MW halo stars, color as in Figure 3. The three stars in Gru II show markedly higher $[\mathrm{Mg} / \mathrm{Ca}]$ ratios than the majority of the comparison stars. The two halo stars with similar abundance signatures, CS 22876-037 and HE 1424-0241 are marked with a black square and diamond, respectively. The star with the highest $[\mathrm{Mg} / \mathrm{Ca}]$ value (orangered dot) is a CEMP-no star in Pis II (Spite et al. 2018).

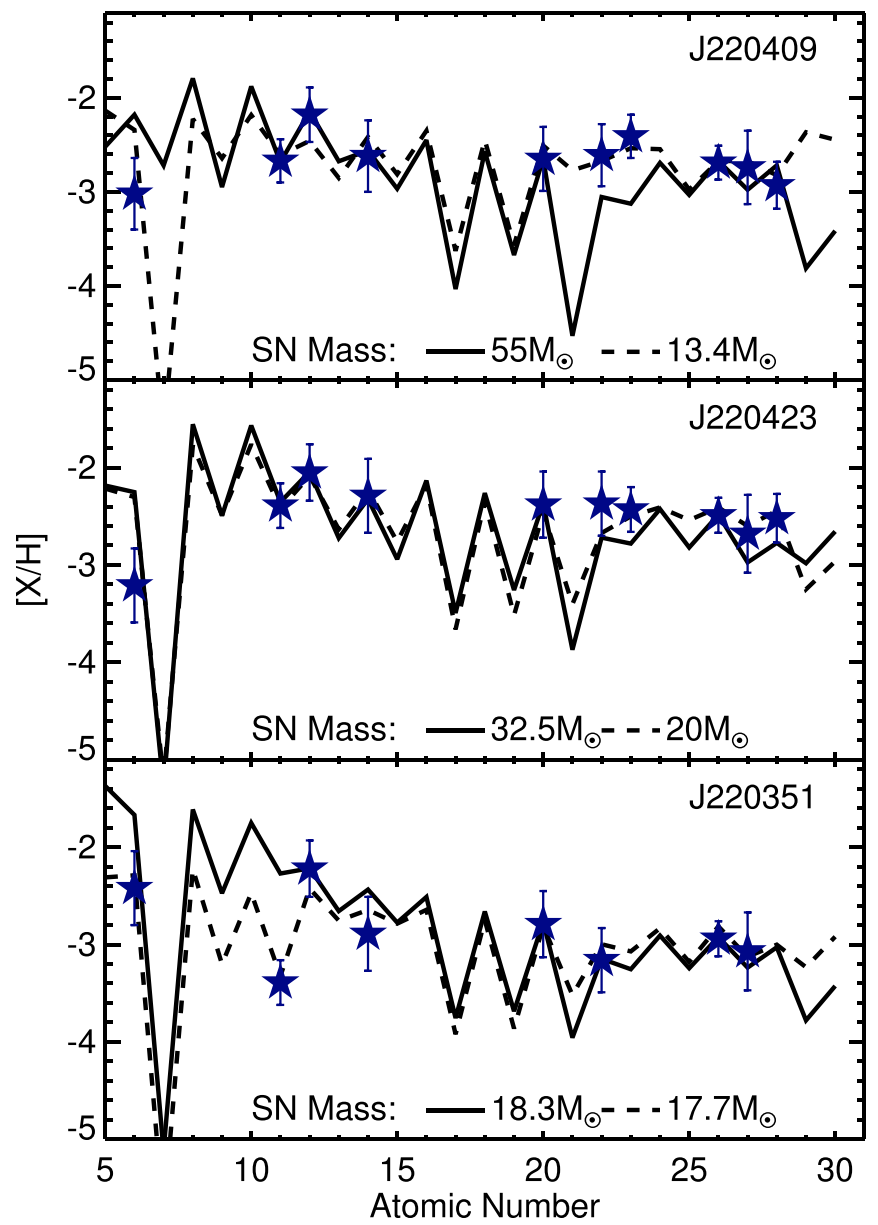

Figure 5. Yields from best-fit SNe models from Heger \& Woosley (2010) to the $[\mathrm{Mg} / \mathrm{H}],[\mathrm{Ca} / \mathrm{H}],[\mathrm{Fe} / \mathrm{H}]$ abundances of the three stars analyzed. Blue stars represent the derived abundances for the three stars. Solid lines show the model fits using only the $[\mathrm{Mg} / \mathrm{H}],[\mathrm{Ca} / \mathrm{H}],[\mathrm{Fe} / \mathrm{H}]$ abundances and dashed lines show model fits using all abundances plotted. number of supernovae. Further determination of $\alpha$-abundances in a larger sample of Gru II stars covering a larger range in metallicity would help to map out the chemical enrichment of this galaxy.

\subsection{Other Alternatives}

An alternative explanation for the low- $\alpha$ abundances detected in the Gru II stars could be an early onset of $\mathrm{SNe}$ Ia. The general chemical evolution scenario predicts an enhancement in $\alpha$ elements at low metallicity as a result of enrichment by CCSNe followed by a downturn or "knee" in $[\alpha / \mathrm{Fe}]$ at the onset of SNe Ia (Tinsley 1979). An early enrichment by SNe Ia was speculated to be the reason for the small $\alpha$-element abundances detected in Hor I (Nagasawa et al. 2018). However, the three stars analyzed here show a "normal" enhancement in $\mathrm{Mg}$ and only low $\mathrm{Si}, \mathrm{Ca}$, and $\mathrm{Ti}$ abundances. An injection of $\mathrm{Fe}$ into the systems would lower all the $[\alpha / \mathrm{Fe}]$ abundance ratios, thus we do not consider this a likely explanation for the $\alpha$-element abundance pattern detected in Gru II. Some UFD galaxies, including Hor I, have also been found to be likely satellites of the Large Magellanic Clouds (LMC) rather than MW satellites (Kallivayalil et al. 2018). It is not yet clear if a different birth environment like the LMC could result in the different chemical abundance patterns seen in some of the LMC-associated UFD galaxies (Nagasawa et al. 2018). However, as a counter-argument, Simon et al. (2020) find it unlikely that Gru II is associated with the LMC.

\subsection{Source of Neutron-capture Elements}

Gru II is the third UFD galaxy in which an enhancement in $r$-process elements has been detected, with Ret II and Tuc III being the other two. The stars in Tuc III and Ret II exhibit a more uniform enhancement in $r$-process elements, with all five stars analyzed in Tuc III showing a mild $r$-process enhancement $([\mathrm{Eu} / \mathrm{Fe}] \sim 0.5)$, and seven of the nine stars in Ret II being extremely enhanced $([\mathrm{Eu} / \mathrm{Fe}]>1)$. In Figure 6 the neutron-capture element abundances in J220423 are compared to the scaled solar system $r$-process residuals from Arlandini et al. (1999). The scaling factor is calculated from the average difference between the J220423 and solar abundances for Ba, $\mathrm{La}, \mathrm{Pr}, \mathrm{Nd}$, and $\mathrm{Eu}$. The neutron-capture abundance pattern in J220423 matches the scaled solar system $r$-process abundance pattern for elements from $\mathrm{Ba}$ to Eu. A similar match has also been found for $r$-process enhanced stars in other ultra-faint and classical dwarf galaxies (Ji et al. 2016c; Hansen et al. 2017; Marshall et al. 2019), suggesting that similar nucleosynthesis events enriched these galaxies. Currently three astrophysical sites for heavy $r$-process element production are proposed; NSM (Lattimer \& Schramm 1974), magnetorotational SN (JetSN) (Winteler et al. 2012), and collapsars (Siegel et al. 2019). Observationally only the NSM has been confirmed to produce $r$-process elements (Drout et al. 2017). Also, more recent models for the Jet-SNe do not find that this site is capable of producing the heavy $r$-process elements (Mösta et al. 2018).

For Ret II, Ji et al. (2016a) argued that the most likely source of $r$-process elements in this galaxy is an NSM, due to the small size of the galaxy and the very large $r$-process element enrichment. It should be noted that collapsars, which could be very efficient $r$-process production sites (Siegel et al. 2019), were only introduced as a possible $r$-process element 


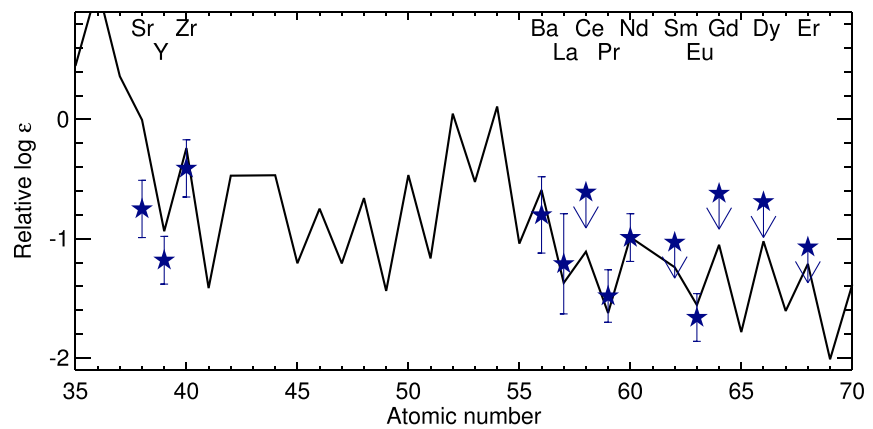

Figure 6. Absolute abundances of neutron-capture elements derived for J220423 compared to the scaled solar system $r$-process abundance pattern from Arlandini et al. (1999).

production site after the $\mathrm{Ji}$ et al. (2016a) study. With the somewhat milder enhancement of Tuc III it was speculated that Tuc III may have been more massive in the past, thus having more gas to dilute the ejecta from the $r$-process nucleosynthesis event compared to Ret II (Hansen et al. 2017; Marshall et al. 2019). This scenario also agrees with the extended tails detected for Tuc III (Li et al. 2018). Alternatively, the gas in Tuc III could also have been polluted by an outside event and thus only received a fraction of the $r$-process ejecta.

When exploring the possible sources for the $r$-process elements detected in Gru II we can consider the possible neutron-capture element production from the progenitors of the $\alpha$-element signature seen in Gru II. For the stars in Her also exhibiting the high $[\mathrm{Mg} / \mathrm{Ca}]$ signature, Koch et al. (2008) determined upper limits of $[\mathrm{Ba} / \mathrm{Fe}]<-2.1$. In fact, Koch et al. (2013) detected a general deficiency of $\mathrm{Ba}$ in a sample of 20 stars in Her. Thus the neutron-capture element enhancement of one of the Gru II stars does not seem to be coupled with the high-mass CCSNe associated with the $\alpha$-element signature detected for some stars in Her and all three stars analyzed in Gru II. In fact, this type of CCSN is likely inefficient in producing neutron-capture elements.

The possible top-heavy nature of the IMF of Gru II, as suggested by the $\alpha$-element abundances of the stars, would result in an increased number of $\mathrm{SNe}$. This in principle increases the chance of all three proposed $r$-process nucleosynthesis sites to occur and does not provide any evidence of which one is most likely to have occurred. Following Ji et al. (2019b) and using the solar $r$-process abundances from Sneden et al. (2008), we calculate the lanthanide fraction $X_{\mathrm{La}}$ for $\mathrm{J} 220423$ and find $\log X_{\mathrm{La}}=-1.2$. This value is very similar to the lanthanide fractions of Tuc III $\left(\log X_{\mathrm{La}}=-1.5\right)$ and Ret II $\left(\log X_{\mathrm{La}}=-1.1\right)(\mathrm{Ji}$ et al. 2019b), and somewhat higher than the value found for GW170817 of $\log X_{\mathrm{La}}=-2.2$. With only one NSM detected to date it is unclear if the lanthanide fraction derived from GW170817 represents the general lanthanide fraction distribution for NSMs; it is therefore difficult to conclude with this number that a NSM is not the source of $r$-process elements in Gru II. The fact that J220409 and J220352, the most metal-poor stars of the three, show no Eu enhancement suggests some delay time from the enrichment of the system with $\alpha$ elements to $r$-process element enrichment, pointing at a NSM as the origin of the $r$-process elements in Gru II. It is also possible that the $r$-process event enriching the gas from which J220423 formed happened in a neighboring system, in which case the lanthanide fraction may be the only clue to the origin of these elements.

\section{Summary}

In this paper we have derived abundances for the three brightest member stars at the top of the giant branch of the UFD galaxy Gru II. High $[\mathrm{Mg} / \mathrm{Ca}]$ ratios were determined for all stars. This abundance signature can be produced via nucleosynthesis in high-mass $\left(\geqslant 20 M_{\odot}\right.$ ) CCSNe (Woosley \& Weaver 1995; Heger \& Woosley 2010), suggesting that Gru II was mainly enriched by a population of very high-mass stars and has a top-heavy IMF. The same chemical signature was also detected in the UFD galaxy Her (Koch et al. 2008), where the low-mass stellar population has been found to have a topheavy IMF (Geha et al. 2013). Alternatively the chemical signature of Gru II can also be the result of stochastic chemical enrichment of the galaxy. To further explore this issue, abundances for a larger sample of stars in Gru II covering a wider metallicity range is needed.

The abundances of Gru II also revealed an enhancement in $r$-process elements in the most metal-rich of the three stars analyzed. This star displays the well-known match to the scaled solar system $r$-process abundance pattern, similar to what has been found previously for other $r$-process enhanced stars in ultra-faint and classical dwarf galaxies and the MW halo (Sneden et al. 2008; Ji et al. 2016c; Hansen et al. 2017). The progenitor of the $r$-process enhancement of Gru II does not seem to be directly coupled with the $\alpha$-element signature. However, the possible top-heavy nature of the IMF of Gru II would result in a larger population of high-mass stars in Gru II and thus also a higher possibility of various types of $\mathrm{SNe}$, leading to $r$-process element production. We calculate a lanthanide fraction of $\log X_{\mathrm{La}}=-1.2$, similar to the fractions found in the other two UFD galaxies with $r$-process enhanced stars, Ret II and Tuc III, and higher than the value derived for GW170817 (Ji et al. 2019b), thus not directly supporting an NSM origin for the $r$-process elements. The possible delay in the $r$-process enhancement compared to the $\alpha$-enhancement of the galaxy, however, does support an NSM as the source of the $r$-process material in this galaxy.

The relationship between the IMF of UFDs and their chemical abundances is relatively unexplored, mainly due to the limited measurements of IMFs of UFD galaxies in combination with the small number of stars in these systems for which abundances can be measured. Expanding this data set will help to better understand the chemical evolution of these systems and the nucleosynthesis of the first stars.

The authors thank the referee for a careful reading of the manuscript and A. McWilliam for useful discussion of abundance error propagation.

Funding for the DES Projects has been provided by the U.S. Department of Energy, the U.S. National Science Foundation, the Ministry of Science and Education of Spain, the Science and Technology Facilities Council of the United Kingdom, the Higher Education Funding Council for England, the National Center for Supercomputing Applications at the University of Illinois at Urbana-Champaign, the Kavli Institute of Cosmological Physics at the University of Chicago, the Center for Cosmology and Astro-Particle Physics at the Ohio State University, the Mitchell Institute for Fundamental Physics and Astronomy at Texas A\&M University, Financiadora de Estudos e Projetos, Fundação Carlos Chagas Filho de Amparo à Pesquisa do Estado do Rio de Janeiro, Conselho Nacional de Desenvolvimento Científico e Tecnológico and the Ministério 
da Ciência, Tecnologia e Inovação, the Deutsche Forschungsgemeinschaft and the Collaborating Institutions in the Dark Energy Survey.

The collaborating institutions are Argonne National Laboratory, the University of California at Santa Cruz, the University of Cambridge, Centro de Investigaciones Energéticas, Medioambientales y Tecnológicas-Madrid, the University of Chicago, University College London, the DES-Brazil Consortium, the University of Edinburgh, the Eidgenössische Technische Hochschule (ETH) Zürich, Fermi National Accelerator Laboratory, the University of Illinois at UrbanaChampaign, the Institut de Ciències de l'Espai (IEEC/CSIC), the Institut de Física d'Altes Energies, Lawrence Berkeley National Laboratory, the Ludwig-Maximilians Universität München and the associated Excellence Cluster Universe, the University of Michigan, the National Optical Astronomy Observatory, the University of Nottingham, The Ohio State University, the University of Pennsylvania, the University of Portsmouth, SLAC National Accelerator Laboratory, Stanford University, the University of Sussex, Texas A\&M University, and the OzDES Membership Consortium.

Based in part on observations at Cerro Tololo InterAmerican Observatory, National Optical Astronomy Observatory, which is operated by the Association of Universities for Research in Astronomy (AURA) under a cooperative agreement with the National Science Foundation.

The DES data management system is supported by the National Science Foundation under grant Nos. AST-1138766 and AST-1536171. The DES participants from Spanish institutions are partially supported by MINECO under grants AYA2015-71825, ESP2015-66861, FPA2015-68048, SEV2016-0588, SEV-2016-0597, and MDM-2015-0509, some of which include ERDF funds from the European Union. I.F.A.E. is partially funded by the CERCA program of the Generalitat de Catalunya. Research leading to these results has received funding from the European Research Council under the European Union's Seventh Framework Program (FP7/20072013) including ERC grant agreements 240672, 291329, and 306478. We acknowledge support from the Brazilian Instituto Nacional de Ciência e Tecnologia (INCT) e-Universe (CNPq grant 465376/2014-2).

T.T.H and J.D.S acknowledge support from NSF grant AST1714873. T.S.L. is supported by NASA through Hubble Fellowship grant HST-HF2-51439.001, awarded by the Space Telescope Science Institute, which is operated by the Association of Universities for Research in Astronomy, Inc., for NASA, under contract NAS5-26555.

This manuscript has been authored by Fermi Research Alliance, LLC under Contract No. DE-AC02-07CH11359 with the U.S. Department of Energy, Office of Science, Office of High Energy Physics.

Facility: Magellan/Clay MIKE.

Software: MOOG (Sneden 1973; Sobeck et al. 2011), IRAF (Tody 1986, 1993), ATLAS9 (Castelli \& Kurucz 2003), linemake (https://github.com/vmplacco/linemake), STARFIT (http://starfit.org/).

\section{ORCID iDs}

T. T. Hansen (ㄷ) https://orcid.org/0000-0001-6154-8983 J. L. Marshall (1) https://orcid.org/0000-0003-0710-9474 T. S. Li (i) https://orcid.org/0000-0002-9110-6163

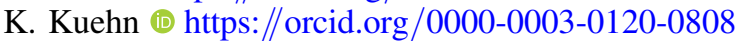

D. Carollo (1) https://orcid.org/0000-0003-4710-132X

M. Geha (1) https://orcid.org/0000-0002-7007-9725

D. James (ㄷ) https://orcid.org/0000-0001-5160-4486

A. Walker (i) https://orcid.org/0000-0002-7123-8943

D. Brooks (i) https://orcid.org/0000-0002-8458-5047

D. L. Burke (i) https://orcid.org/0000-0003-1866-1950

M. Carrasco Kind (1) https://orcid.org/0000-0002-4802-3194

K. Eckert (1) https://orcid.org/0000-0002-1407-4700

J. García-Bellido (1) https://orcid.org/0000-0002-9370-8360

D. W. Gerdes (10) https://orcid.org/0000-0001-6942-2736

D. Gruen (1) https://orcid.org/0000-0003-3270-7644

R. A. Gruendl (1) https://orcid.org/0000-0002-4588-6517

S. R. Hinton (1) https://orcid.org/0000-0003-2071-9349

D. L. Hollowood (1) https://orcid.org/0000-0002-9369-4157

R. Miquel (i) https://orcid.org/0000-0002-6610-4836

A. Palmese (1) https://orcid.org/0000-0002-6011-0530

F. Paz-Chinchón (10) https://orcid.org/0000-0003-1339-2683

A. A. Plazas (i) https://orcid.org/0000-0002-2598-0514

E. Sanchez (i) https://orcid.org/0000-0002-9646-8198

M. Smith (i) https://orcid.org/0000-0002-3321-1432

M. Soares-Santos (1) https://orcid.org/0000-0001-6082-8529

M. E. C. Swanson (1) https://orcid.org/0000-0002-1488-8552

G. Tarle (i) https://orcid.org/0000-0003-1704-0781

\section{References}

Abbott, T. M. C., Abdalla, F. B., Allam, S., et al. 2018, ApJS, 239, 18 Aoki, W., Beers, T. C., Christlieb, N., et al. 2007, ApJ, 655, 492

Arlandini, C., Käppeler, F., Wisshak, K., et al. 1999, ApJ, 525, 886 Asplund, M., Grevesse, N., Sauval, A. J., et al. 2009, ARA\&A, 47, 481 Barklem, P. S., Christlieb, N., Beers, T. C., et al. 2005, A\&A, 439, 129 Belokurov, V., Zucker, D. B., Evans, N. W., et al. 2007, ApJ, 654, 897 Bernstein, R., Shectman, S. A., Gunnels, S. M., Mochnacki, S., \& Athey, A. E. 2003, Proc. SPIE, 4841, 1694

Casagrande, L., Ramírez, I., Meléndez, J., et al. 2010, A\&A, 512, A54

Castelli, F., \& Kurucz, R. L. 2003, in IAU Symp. 210, Modelling of Stellar Atmospheres (Cambridge: Cambridge Univ. Press), A20

Cayrel, R. 1988, in IAU Symp. 132, The Impact of Very High S/N Spectroscopy on Stellar Physics (Dordrecht: Kluwer), 345

Chiti, A., Frebel, A., Ji, A. P., et al. 2018, ApJ, 857, 74

Chubak, C., Marcy, G., Fischer, D. A., et al. 2012, arXiv:1207.6212

Cohen, J. G., McWilliam, A., Christlieb, N., et al. 2007, ApJL, 659, L161

Dotter, A., Chaboyer, B., Jevremović, D., et al. 2008, ApJS, 178, 89

Drlica-Wagner, A., Bechtol, K., Rykoff, E. S., et al. 2015, ApJ, 813, 109

Drlica-Wagner, A., Sevilla-Noarbe, I., Rykoff, E. S., et al. 2018, ApJS, 235, 33

Drout, M. R., Piro, A. L., Shappee, B. J., et al. 2017, Sci, 358, 1570

Feltzing, S., Eriksson, K., Kleyna, J., et al. 2009, A\&A, 508, L1

François, P., Monaco, L., Bonifacio, P., et al. 2016, A\&A, 588, A7

Frebel, A., Casey, A. R., Jacobson, H. R., et al. 2013, ApJ, 769, 57

Frebel, A., \& Norris, J. E. 2015, ARA\&A, 53, 631

Frebel, A., Norris, J. E., Gilmore, G., et al. 2016, ApJ, 826, 110

Frebel, A., Simon, J. D., Geha, M., et al. 2010, ApJ, 708, 560

Frebel, A., Simon, J. D., \& Kirby, E. N. 2014, ApJ, 786, 74

Geha, M., Brown, T. M., Tumlinson, J., et al. 2013, ApJ, 771, 29

Gennaro, M., Tchernyshyov, K., Brown, T. M., et al. 2018, ApJ, 855, 20

Gilmore, G., Norris, J. E., Monaco, L., et al. 2013, ApJ, 763, 61

Hansen, T. T., Holmbeck, E. M., Beers, T. C., et al. 2018, ApJ, 858, 92

Hansen, T. T., Simon, J. D., Marshall, J. L., et al. 2017, ApJ, 838, 44

Heger, A., \& Woosley, S. E. 2010, ApJ, 724, 341

Ishigaki, M. N., Aoki, W., Arimoto, N., et al. 2014, A\&A, 562, A146

Ji, A. P., Drout, M. R., \& Hansen, T. T. 2019a, ApJ, 882, 40

Ji, A. P., Frebel, A., Chiti, A., et al. 2016a, Natur, 531, 610

Ji, A. P., Frebel, A., Ezzeddine, R., et al. 2016b, ApJL, 832, L3

Ji, A. P., Frebel, A., Simon, J. D., et al. 2016c, ApJ, 830, 93

Ji, A. P., Frebel, A., Simon, J. D., et al. 2016d, ApJ, 817, 41

Ji, A. P., Li, T. S., Simon, J. D., et al. 2020, ApJ, 889, 27

Ji, A. P., Simon, J. D., Frebel, A., et al. 2019b, ApJ, 870, 83

Johnson, J. A. 2002, ApJS, 139, 219

Kalirai, J. S., Anderson, J., Dotter, A., et al. 2013, ApJ, 763, 110

Kallivayalil, N., Sales, L. V., Zivick, P., et al. 2018, ApJ, 867, 19

Kelson, D. D. 2003, PASP, 115, 688 
Kelson, D. D., Illingworth, G. D., van Dokkum, P. G., et al. 2000, ApJ, 531, 159

Koch, A., Feltzing, S., Adén, D., et al. 2013, A\&A, 554, A5

Koch, A., McWilliam, A., Grebel, E. K., et al. 2008, ApJL, 688, L13

Kroupa, P. 2001, MNRAS, 322, 231

Lattimer, J. M., \& Schramm, D. N. 1974, ApJL, 192, L145

Li, T. S., Simon, J. D., Kuehn, K., et al. 2018, ApJ, 866, 22

Maeder, A., Meynet, G., \& Chiappini, C. 2015, A\&A, 576, A56

Marshall, J. L., Hansen, T., Simon, J. D., et al. 2019, ApJ, 882, 177

McWilliam, A., Preston, G. W., Sneden, C., et al. 1995, AJ, 109, 2757

McWilliam, A., \& Searle, L. 1999, Ap\&SS, 265, 133

McWilliam, A., Wallerstein, G., \& Mottini, M. 2013, ApJ, 778, 149

Mösta, P., Roberts, L. F., Halevi, G., et al. 2018, ApJ, 864, 171

Nagasawa, D. Q., Marshall, J. L., Li, T. S., et al. 2018, ApJ, 852, 99

Norris, J. E., Beers, T. C., \& Ryan, S. G. 2000, ApJ, 540, 456

Norris, J. E., Wyse, R. F. G., Gilmore, G., et al. 2010, ApJ, 723, 1632

Pace, A. B., \& Li, T. S. 2019, ApJ, 875, 77

Placco, V. M., Frebel, A., Beers, T. C., et al. 2014, ApJ, 797, 21

Placco, V. M., Frebel, A., Beers, T. C., et al. 2016, ApJ, 833, 21

Roederer, I. U., \& Kirby, E. N. 2014, MNRAS, 440, 2665
Roederer, I. U., Mateo, M., Bailey, J. I., et al. 2016, AJ, 151, 82

Roederer, I. U., Preston, G. W., Thompson, I. B., et al. 2014, AJ, 147, 136

Salpeter, E. E. 1955, ApJ, 121, 161

Siegel, D. M., Barnes, J., \& Metzger, B. D. 2019, Natur, 569, 241

Simon, J. D. 2019, ARA\&A, 57, 375

Simon, J. D., Frebel, A., McWilliam, A., et al. 2010, ApJ, 716, 446

Simon, J. D., Li, T. S., Erkal, D., et al. 2020, ApJ, 892, 137

Sneden, C., Cowan, J. J., \& Gallino, R. 2008, ARA\&A, 46, 241

Sneden, C. A. 1973, PhD thesis, Univ. of Texas at Austin

Sobeck, J. S., Kraft, R. P., Sneden, C., et al. 2011, AJ, 141, 175

Spite, M., Spite, F., François, P., et al. 2018, A\&A, 617, A56

Tinsley, B. M. 1979, ApJ, 229, 1046

Tody, D. 1986, Proc. SPIE, 627, 733

Tody, D. 1993, in ASP Conf. Ser. 173, Astronomical Data Analysis Software and Systems II, ed. R. J. Hanisch, R. J. V. Brissenden, \& J. Barnes (San Francisco, CA: ASP), 173

Tonry, J., \& Davis, M. 1979, AJ, 84, 1511

Umeda, H., \& Nomoto, K. 2003, Natur, 422, 871

Winteler, C., Käppeli, R., Perego, A., et al. 2012, ApJL, 750, L22

Woosley, S. E., \& Weaver, T. A. 1995, ApJS, 101, 181 